Amino Acids (1992) 2: 25-67

$\overline{\text { Acids }}$ mino

(C) Springer-Verlag 1992
Printed in Austria

\title{
Differential diagnosis of (inherited) amino acid metabolism or transport disorders
}

\author{
W. Blom and J. G. M. Huijmans \\ Metabolic Laboratory, Department of Pediatrics, Sophia Children's Hospital, Erasmus \\ University Rotterdam, The Netherlands
}

Summary. Disorders of amino acid metabolism or transport are most clearly expressed in urine. Nevertheless the interpretation of abnormalities in urinary amino acid excretion remains difficult. An increase or decrease of almost every amino acid in urine can be due to various etiology. To differentiate between primary and secondary aminoacido-pathies systematic laboratory investigation is necessary. Early diagnosis of disorders of amino acid metabolism or transport is very important, because most of them can be treated, leading to the prevention of (further) clinical abnormalities. In those disorders, which cannot be treated, early diagnosis in an index-patient may prevent the birth of other siblings by means of genetic counseling and prenatal diagnosis.

Primary aminoacidopathies can be due to genetically determined transport disorders and enzyme deficiencies in amino acid metabolism or degradation. Secondary aminoacidopathies are the result of abnormal or deficient nutrition, intestinal dysfunction, organ pathology or other metabolic diseases like organic acidurias.

A survey of amino acid metabolism and transport abnormalities will be given, illustrated with metabolic pathways and characteristic abnormal amino acid chromatograms.

Keywords: Amino acids - Inborn errors of metabolism

\section{Introduction}

Like other inborn errors of metabolism the disorders of amino acid metabolism are caused by a single enzyme deficiency or a transport abnormality. The clinical expression of amino acid metabolism or transport disorders has a great variety. The course of the disease can be acute, chronic or intermittent, but the clinical symptoms can even be masked by multifactorial medical problems.

Fig. 1 schematically shows the physiology of amino acids. Amino acids need to be transported over the intestinal wall, the cell and cell-organelle 


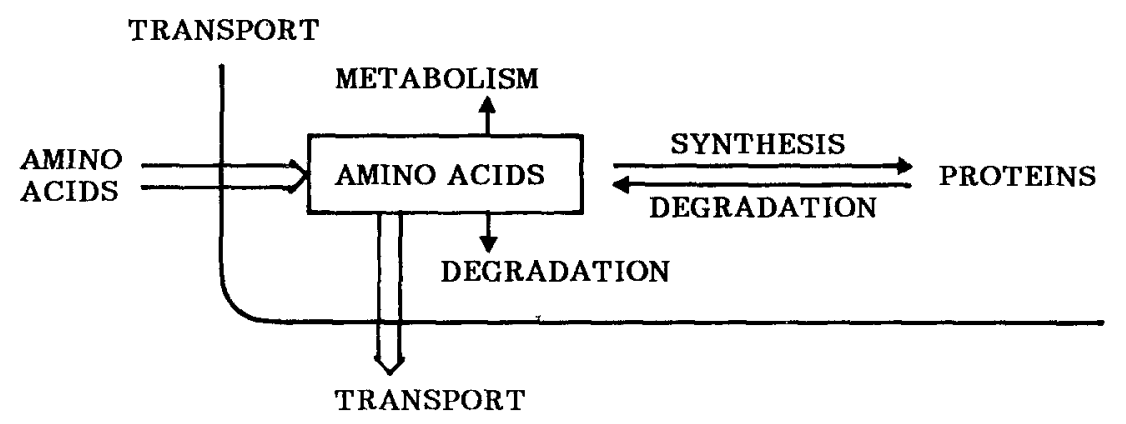

Fig. 1. Scheme for amino acid transport, metabolism, and degradation

membranes, the blood-brain barrier, the renal tubular wall, etc. For some amino acids there are special active transport mechanisms. A number of amino acids are sharing the same special transport mechanism (e.g. proline, hydroxyproline and glycine, or cystine, ornithine, lysine and arginine). Other amino acids are in competition for their transport (e.g. the branched-chain amino acids, phenylalanine, tyrosine and tryptophan). Insuline stimulates cellular uptake of amino acids, except tryptophan, which is mainly bound to albumin.

Normally there is an equilibrium between synthesis and degradation of proteins with respectively, consumption and liberation of amino acids. Amino acids can be metabolized into other amino acids, hormones, neurotransmitters, pigments, etc. The excess of amino acids can be degraded, mostly into organic acids. The first degradation step is usually the removal of nitrogen by transamination or deamination.

Protein and amino acid metabolism is under hormonal regulation and depends on the nutritional state. In the anabolic state, amino acid metabolism and protein synthesis are enhanced, whereas in the catabolic state, amino acid and protein degradation dominates.

Some enzymes involved in amino acid metabolism or degradation need coenzymes or cofactors, which means that vitamins, trace elements, etc. may play an important role in the enzymatic action.

All amino acid metabolism or transport disorders are expressed in urine by accumulation of one or more amino acids, sometimes in combination with a decrease in the urinary excretion of one or more other amino acids. The interpretation of the amino acid chromatogram is not always that simple. Often, additional laboratory investigations are necessary; at least amino acid analysis in plasma, and in rare cases also in cerebrospinal fluid, has to be performed. Also other chromatographic techniques need to be used, e.g. gas chromatography (+ mass spectrometry) of organic acids, thin-layer chromatography of imidazoles, indoles and sugars. The estimation of single metabolites as $\mathrm{NH}_{3}$, orotic acid, methylmalonic acid, oxalic acid, etc. might be necessary. For prenatal diagnosis, amino acid analysis in amniotic fluid can be a solution. To confirm a diagnosis, enzyme analysis in tissue (e.g. liver), blood cells, or cultured skin fibroblast is very often desirable or necessary. However, the most powerful new technique for studying human chemical genetics are those of recombinant DNA technology. 


\section{Methods}

Amino acid analysis

Amino acid analysis was performed with an LKB 4151 Alpha Plus ${ }^{\circledR}$ Amino Acid Analyser. Urines were deproteinized by mixing $500 \mu \mathrm{l}$ of urine with $500 \mu \mathrm{l}$ of a $5 \%$ sulfosalicylic acid solution containing $0,5 \mathrm{mmol} / 1$ norleucine (Nleu) as internal standard. The mixture was centrifuged for $2 \mathrm{~min}$ at $10,000 \mathrm{rpm}$. The volume of sample loaded on the ion-exchange column was $40 \mu \mathrm{l}$ of the deproteinized supernatant. For analysis of amino acids we used the standard stepwise elution by five lithium-citrate buffers through a column containing $8 \mu \mathrm{m}$ cation-exchange resin. The amino acids were detected with ninhydrin reagent through a reaction coil set at $135^{\circ} \mathrm{C}$. The O.D. range/gain was 1.0 for both the $440 \mathrm{~nm}$ and $570 \mathrm{~nm}$ absorbance. In the chromatograms shown, the $440 \mathrm{~nm}$ absorbance is the lower signal and the $570 \mathrm{~nm}$ absorbance the upper signal. The recorder speed was $2 \mathrm{~mm} / \mathrm{min}$.

\section{Organic acid analysis}

Organic acids in urine were estimated as described elsewhere [1].

\section{Aminoacidopathies - Results and discussion}

For general information and a guidance for references to disorders of amino acid metabolism and transport we refer to a few handbooks [2-6]. Normal values are extensively given in Bremer et al. [2]. In the text we refer to the cataloque items of McKusick [7].

In Fig. 2 the picture of a normal chromatogram of amino acids in urine is given. We mostly use norleucine (NLEU) as an internal standard. Abnormal amino acid chromatograms can be compared with Fig. 2.

The sequence of amino acid elution is only slightly variable for different amino acid analysers. For that reason we want to present and to discuss the results of amino acid analysis in the elution-sequence of the amino acids in the chromatogram.

\section{Taurine}

A primary metabolic disorder in taurine metabolism is not described. Secondarily urinary taurine excretion can be increased in a catabolic state, during a high protein intake, as a result of acute liver failure, and in sulphite oxidase deficiency.

\section{Phosphoethanolamine}

In hypophosphatasia due to a deficiency of tissue-nonspecific (liver, bone, kidney) alkaline phosphatase isoenzyme (ALPL) phosphoethanolamine is accumulating in urine. (McKusick 14630, 24150, 24151). Besides phosphoethanolamine also pyrophosphate and pyridoxal-5'-phosphate are accumulating. Hypophosphatasia causes defective skeletal mineralization in infants and children, and osteomalacia in adults. In the perinatal form severe skeletal abormalities can occur in utero leading to stillbirth. The infantile form can be associated 


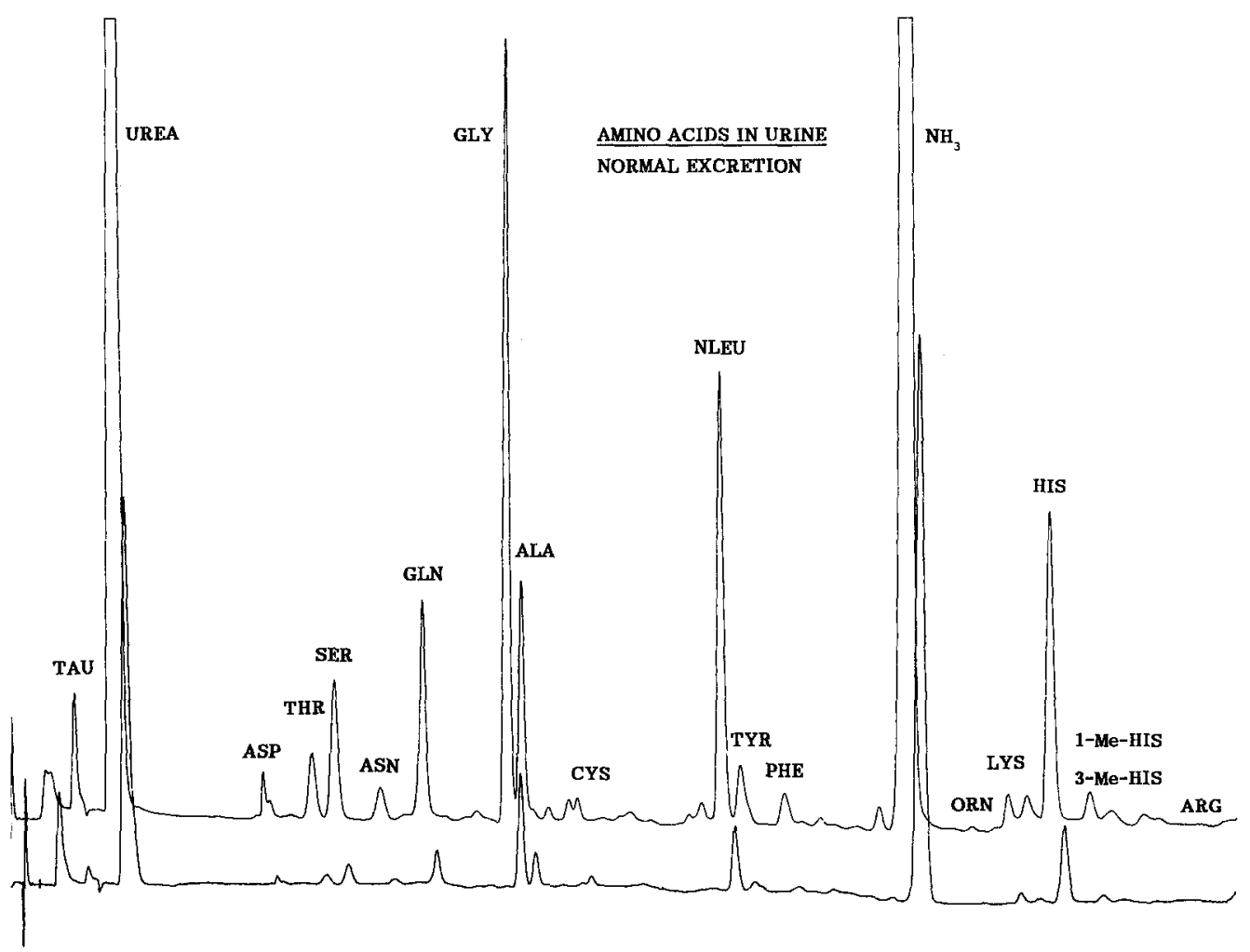

Fig. 2. Normal amino acid chromatogram of urine

with nephrocalcinosis from hypercalcemia and might be fatal. At different ages the clinical expression can be very variable.

Secondarily phosphoethanolamine excretion in urine can occur in some endocrine disorders, in different bone diseases, and as a result of hypertension.

\section{Aspartylglycosamine}

Aspartylglycosamine is a ninhydrin-positive metabolite, detectable in substantial amounts in aspartylglycosaminuria due to lysosomal aspartylglycosaminidase deficiency. (McKusick 20840) (see Fig. 3). The clinical phenotype of patients with aspartylglycosaminuria resemble that of mild mucopolysaccharidosis with progressive mental deterioration. The disease is mainly diagnosed in Finland.

The laboratory diagnosis can also be made by thin-layer chromatography of oligosaccharides (unless the urine is desalted with ion-exchange resins before analysis).

\section{Aspartic acid}

Aspartic aciduria together with glutamic aciduria was described in dicarboxylic aminoaciduria, a renal tubular transport disorder associated with hyperprolinuria. (McKusick 22273). Only two patients are reported. Aspartic acid can also arise from the decomposition of asparagine. 

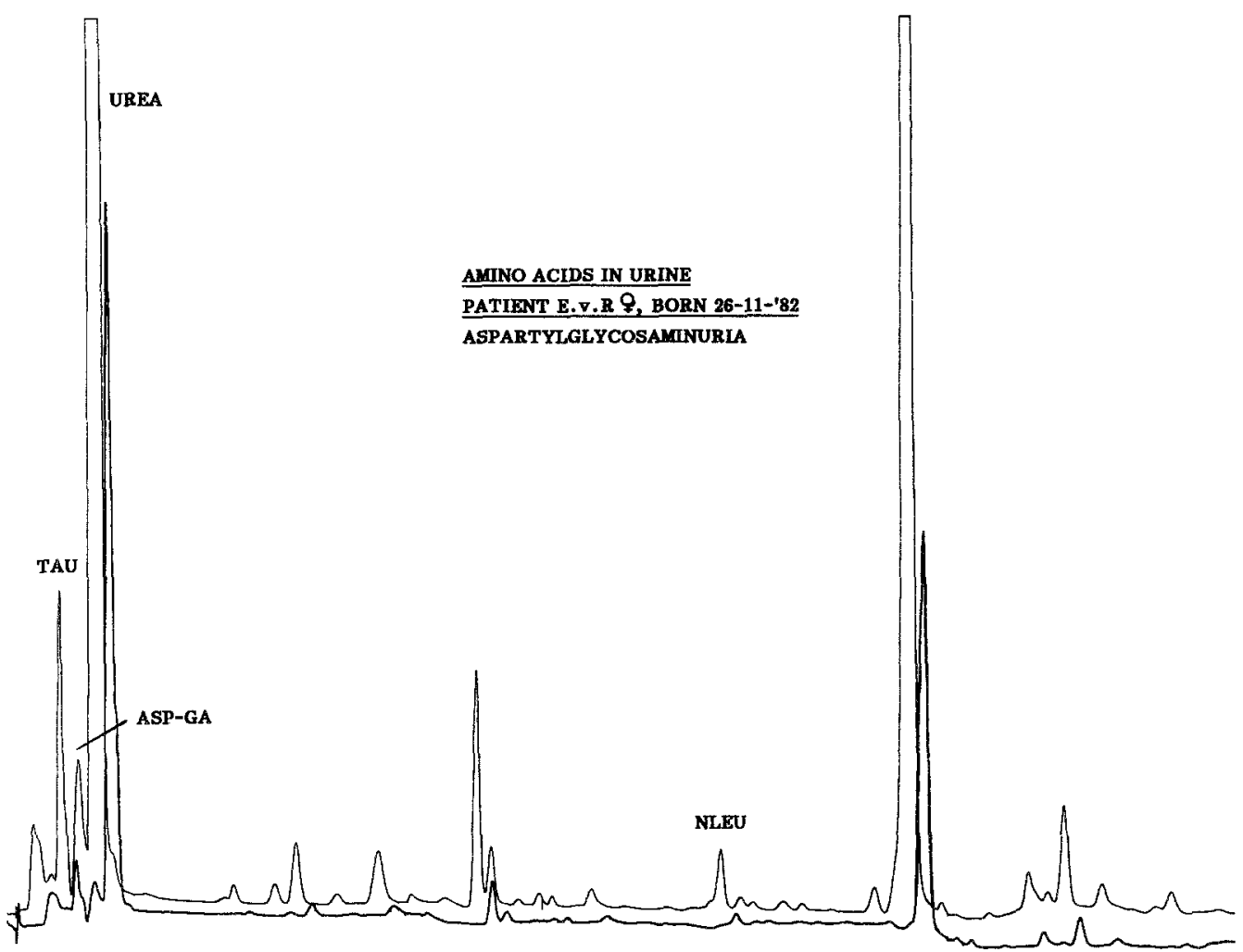

Fig. 3. Chromatogram of amino acids in urine from a patient with lysosomal aspartylglycosylaminase deficiency. The urine is diluted $8 x$

\section{4-Hydroxyproline}

In 4-hydroxy-L-proline oxidase deficiency hydroxyproline concentration in plasma and urine is increased. (McKusick 23700). Mental retardation and microscopic hematuria are clinical features, although patients have been reported without clinical abnormalities.

Hydroxyprolinuria is also seen in iminoglycinuria (McKusick 24260), a benign inborn error of amino acid transport, affecting the renal tubular reabsorptive mechanism of glycine, proline and hydroxyproline.

Hydroxyproline excretion in urine is increased in newborns, in chronic uraemia, in liver cirrhosis and in some bone diseases.

\section{Threonine and serine}

Primary disorders of threonine and serine metabolism are not described. Both hydroxy-amino acids can be increased in vitamine $B_{6}$ deficiency. The concentration of threonine and serine in urine rapidly diminish by bacterial decomposition of a urine. Threonine can be increased in liver cirrhosis. Some infant formulas are leading to an increased concentration of threonine in plasma and urine. Urinary serine excretion can also be diminished in folic acid deficiency. 


\section{Asparagine}

Disorders in asparagine metabolism are not described. Urinary asparagine excretion is elevated in hyperammonemia, and in Hartnup disease.

\section{Glutamic acid}

Glutamic aciduria together with aspartic aciduria and hyperprolinuria is a renal tubular transport disorder already mentioned under 4. aspartic acid (McKusick 22273).

Only one patient with persistent glutamic acidemia was reported in the literature [8]. The single patient showed failure to thrive, vomiting, diarrhea, and acidosis, which persisted despite dietary treatment. A sibling had died at the age of 7 weeks with a similar clinical picture.

Glutamic acid can secondarily be increased in urine by decomposition of glutamine.

\section{Glutamine}

A primary metabolic defect in glutamine metabolism is not described. Glutamine is secondarily increased in all conditions with hyperammonemia together with alanine. See also Fig. 16. In acutely sick newborns urinary glutamine excretion can be increased.

\section{Sarcosine}

In sarcosine dehydrogenase deficiency sarcosine is strongly elevated in plasma and urine. (McKusick 26890). Sarcosine dehydrogenase is a mitochondrial en-

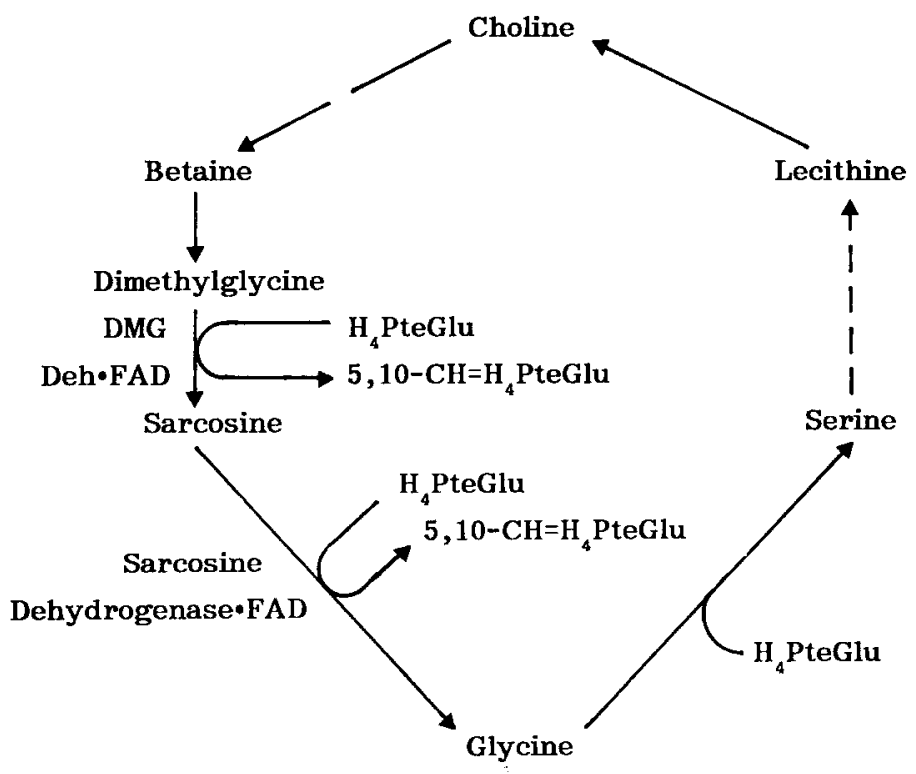

Fig. 4. Metabolic pathway of sarcosine metabolism 
zyme only expressed in liver and kidney, which activates the conversion of sarcosine (=N-methylglycine) into glycine (see Fig. 4). Sarcosine dehydrogenase contains covalently bound FAD and dissociable folate cofactors. Electron transfer from the enzyme to the main respiratory chain is mediated by ETF (electron transport flavoprotein) and an ETF dehydrogenase: ETF-QO (electron transport flavoprotein-ubiquinone oxidoreductase) (see Fig. 5).

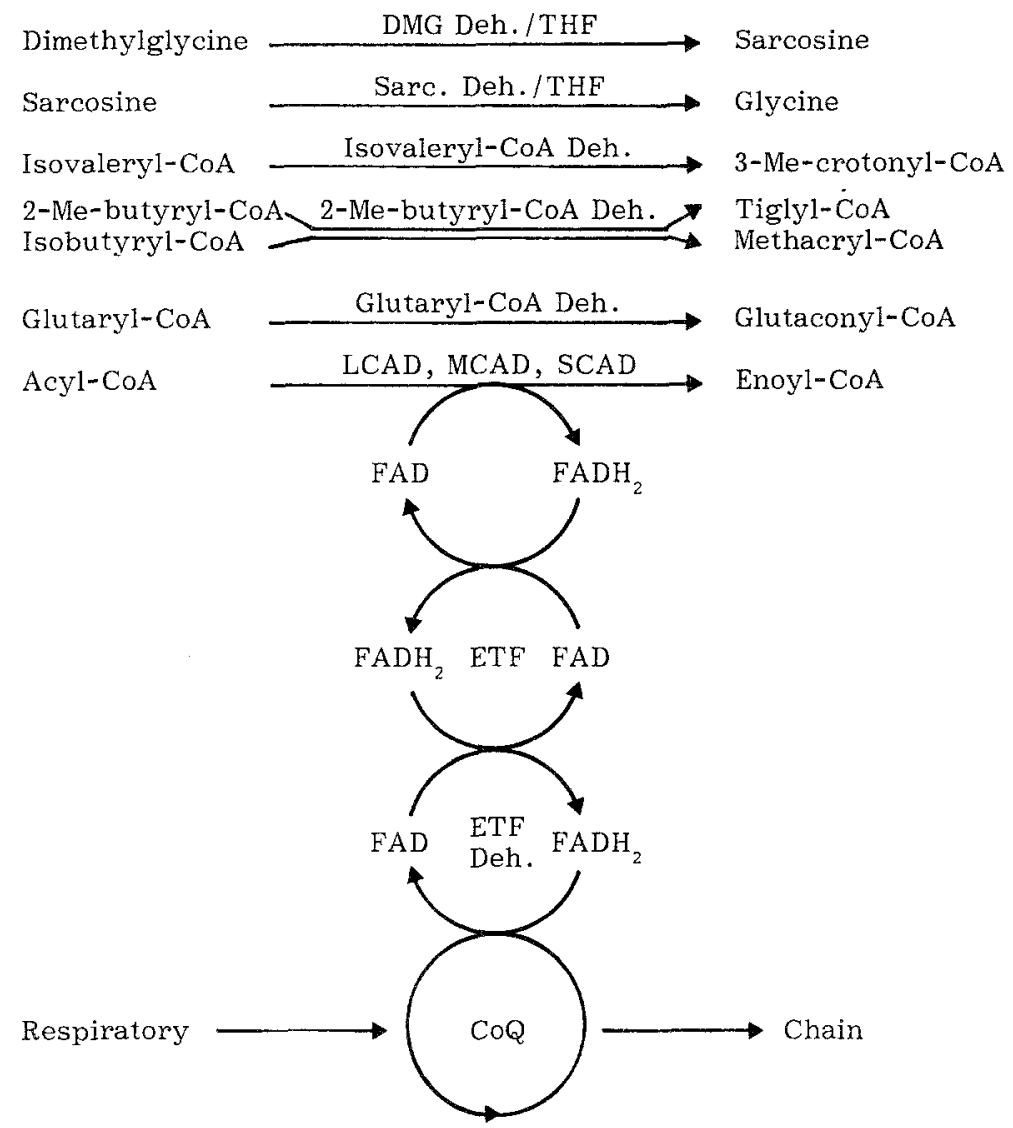

Fig. 5. Electron transport of mitochondrial dehydrogenase reactions to the respiratory chain

The phenotype of sarcosinemia or sarcosinuria can occur on the basis of at least three mechanisms:

1. A defect in the holoenzyme of sarcosine dehydrogenase (e.g. apoenzyme deficiency, or binding defect of folate cofactors to the apoenzyme).

2. A mutation in the electron transport flavoprotein, that is shared by sarcosine dehydrogenase, dimethylglycine dehydrogenase, and other acyl-CoA dehy- 
drogenases (e.g. ETF or ETF-QO deficiency, leading to glutaric aciduria type II).

3. Severe folate deficiency.

The clinical picture of hypersarcosinemia patients is diverse. Some patients have mental retardation, growth delay, vomiting, hypertension, cranial stenosis, syndactyly, and are hypoactive. Another group of patients has only mild mental retardation. A third group of patients has no mental retardation, but is vomiting with growth retardation, and has dyslexia and an enlarged liver. The difference in clinical expression might be due to a variety of genetical mutations.

Fig. 6 shows the amino acid chromatogram in urine from a hypersarcosinemic patient, in which folic acid dependency was discovered [9].

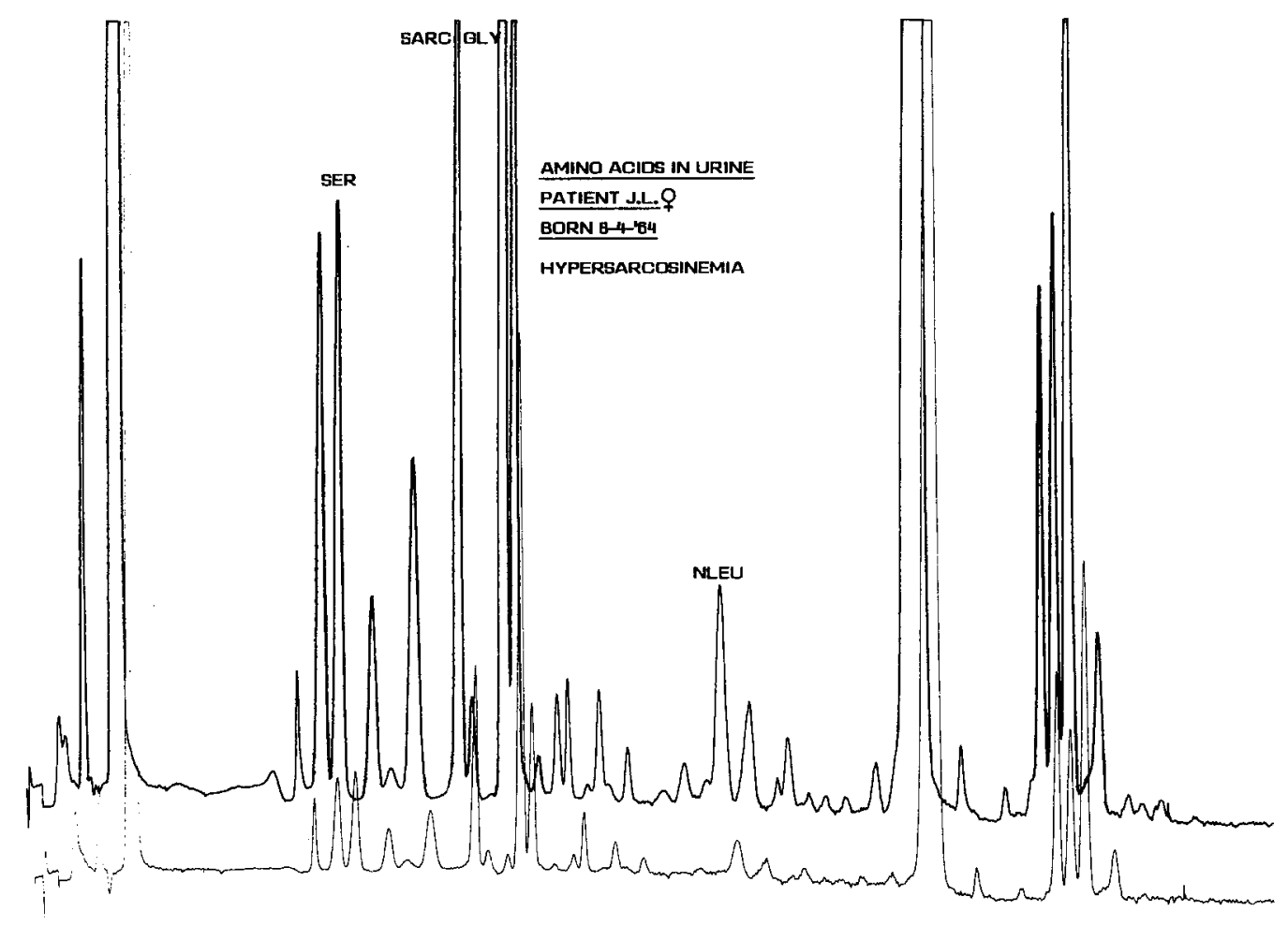

Fig. 6. Chromatogram of amino acids in urine from a patient with sarcosine dehydrogenase deficiency

\section{1. $\alpha$-Aminoadipic acid}

$\alpha$-Aminoadipic acid is normally transaminated into $\alpha$-ketoadipic acid, which is converted into glutaryl-CoA catalyzed by $\alpha$-ketoadipic acid dehydrogenase (see Fig. 7). In 1974 Fisher et al. [10] described the first two patients with $\alpha$-aminoadipic aciduria. (McKusick 20475). A deficiency of the enzyme $\alpha$-aminoadipic acid transaminase was postulated. Later in 1980 Fisher and Brown [11] discovered that these patients were also excreting high amounts of $\alpha$ ketoadipic acid in their urine. In the mean time other patients were reported with $\alpha$-ketoadipic aciduria with an enzymatically proven $\alpha$-ketoadipic acid 
dehydrogenase deficiency (McKusick 24513). The transamination reaction is reversible, which means that in $\alpha$-ketoadipic aciduria not only $\alpha$-ketoadipic acid will accumulate, but also $\alpha$-aminoadipic acid. In our opinion all patients reported with $\alpha$-aminoadipic aciduria are patients with $\alpha$-ketoadipic aciduria due to a deficiency of $\alpha$-ketoadipic acid dehydrogenase.

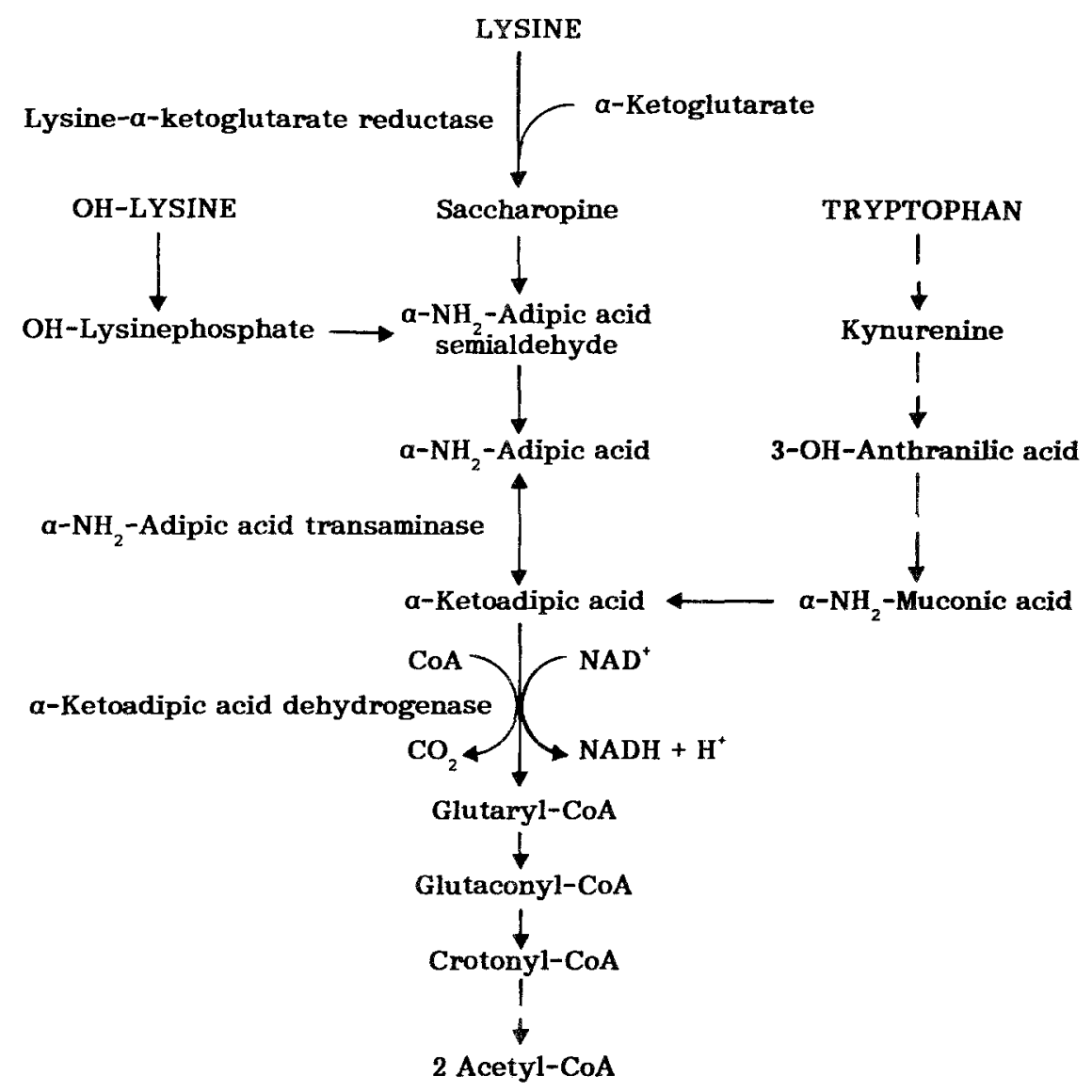

Fig. 7. Degradation pathway of lysine in relation to $\mathrm{OH}-\mathrm{lysine}$ and tryptophan breakdown

Fig. 8 shows the amino acid chromatogram of a patient with $\alpha$-aminoadipic aciduria. If in the same urine organic acid analysis is performed, a significant abnormal peak of $\alpha$-ketoadipic acid can be detected (see Fig. 9). The patient is consequently suffering from $\alpha$-ketoadipic acid dehydrogenase deficiency.

$\alpha$-Ketoadipic acid dehydrogenase deficiency is an inborn error of lysine, tryptophan and hydroxylysine metabolism, which may have no clinical significance. Because, besides patients with psychomotor retardation and neurological abnormalities, cases are described with $\alpha$-ketoadipic aciduria, but without clinical abnormalities.

We observed mild $\alpha$-aminoadipic aciduria in patients with severe seizures. After normalization of the convulsive condition the excretion of $\alpha$-aminoadipic acid also normalized. We do not have any explanation for this phenomenon. 


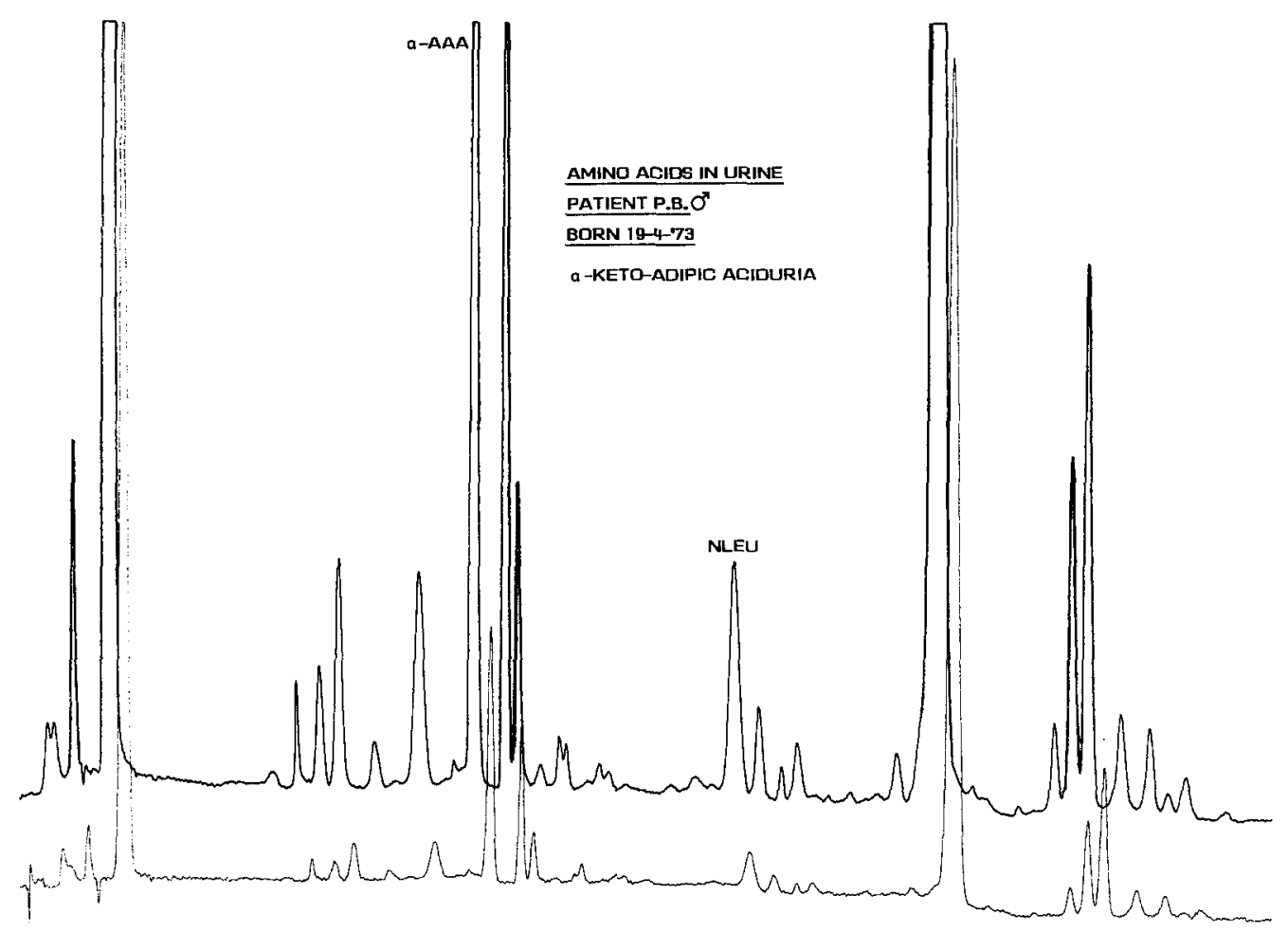

Fig. 8. Chromatogram of amino acids in urine from a patient with $\alpha$-ketoadipic acid dehydrogenase deficiency

\section{Proline}

Two different types of inherited hyperprolinemias exist. In hyperprolinemia type I proline oxidase is deficient. (McKusick 23950) (see step 1 in Fig. 10). Besides hyperprolinuria a persistent hyperprolinemia ( $>450 \mu \mathrm{mol} / 1$, but $<2000$ $\mu \mathrm{mol} / 1)$ is characteristic for the diagnosis. Within the families of hyperprolinemia type I, patients with different forms of ocular disease, mental retardation, and other neurologic manifestations have been described, but these clinical abnormalities were not shared by all probands. Therefore hyperprolinemia type $I$ is considered as a benign disorder.

In hyperprolinemia type II the enzyme L- $\Delta^{1}$-pyrroline-5-carboxylic acid dehydrogenase is deficient. (McKusick 23951). See step 5 in Fig. 10. Hyperprolinemia type II can be distinguished from type I by the much higher plasma proline concentrations $(>2000 \mu \mathrm{mol} / 1)$, and the elevated urinary excretion of L- $\Delta^{\mathbf{1}}$-pyrroline-5-carboxylic acid (P5C) and L- $\Delta^{1}$-pyrroline-3-hydroxy-5carboxylic acid. Not all patients with type II hyperprolinemia have clinical abnormalities, but in symptomatic patients seizures and mental retardation predominate. Type II hyperprolinemia might also be a benign metabolic disorder.

In both types of hyperprolinemia plasma proline is elevated. If the plasma proline concentration exceeds $800 \mu \mathrm{mol} / 1$, which is mostly the case in hyperprolinemia type II, urinary hydroxyproline and glycine concentrations are also elevated. Glycine and hydroxyproline share a transport carrier with proline. 


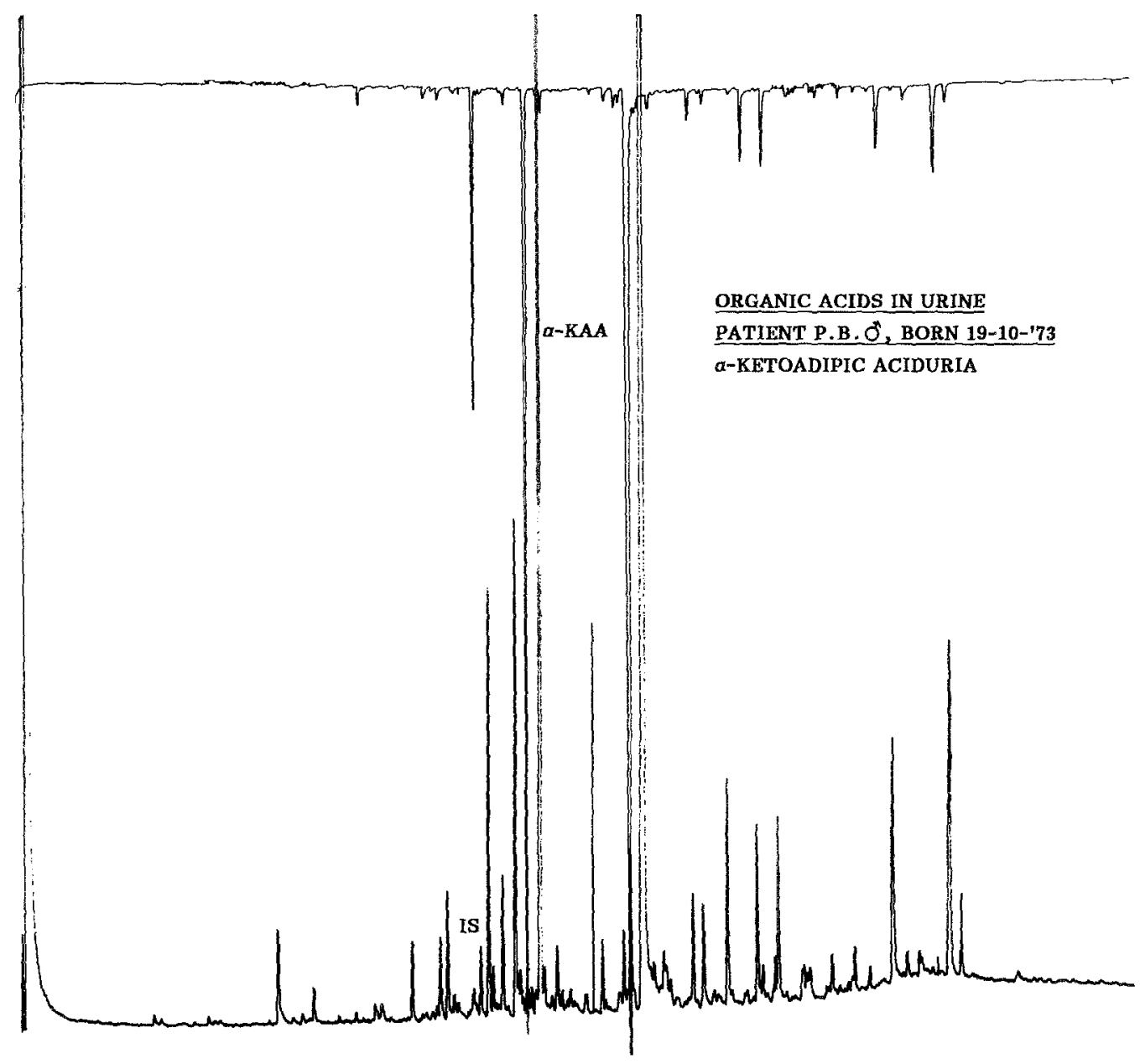

Fig. 9. Gas chromatogram of organic acids in urine from a patient with $\alpha$-ketoadipic acid dehydrogenase deficiency. $\alpha$-Ketoadipic acid is derivatized as ethoxime and methylester

When the concentration of proline is too high in the renal filtrate, reabsorption of hydroxyproline and glycine are competitively inhibited, and "iminoglycinuria" occurs.

In some cases of imidodipeptiduria due to prolidase deficiency (McKusick 26413) proline and hydroxyproline excretion in urine can be increased (besides many proline-containing peptides). Proline excretion is mostly elevated in newborn infants.

\section{Glycine}

A primary disorder of glycine metabolism has appeared in the literature as nonketotic hyperglycinemia (McK usick 23830, 23831 and 23833). The molecular defect lies in the glycine cleavage system, a multienzyme complex with four protein components (see Fig. 11). These protein components are referred to as the $\mathrm{P}$ protein, $\mathrm{H}$ protein, $\mathrm{T}$ protein and $\mathrm{L}$ protein. The $\mathrm{P}$ protein is a pyridoxalphosphate dependent glycine decarboxylase. The $\mathrm{H}$ protein is a lipoic 


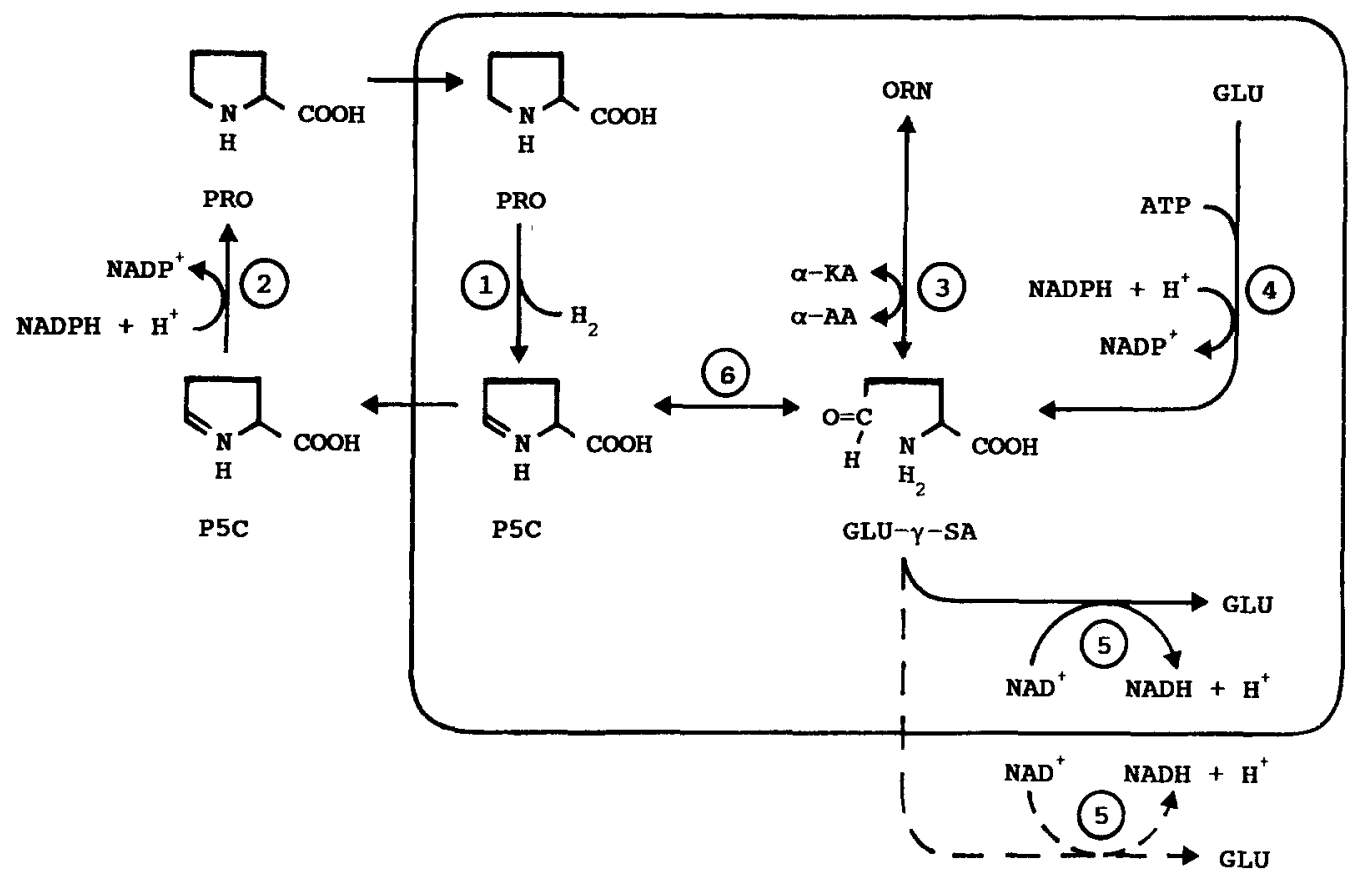

Fig. 10. Metabolic pathway of proline metabolism. The rectangle indicates a mitochondrion. P5C L- $\Delta^{1}$-pyrroline-5-carboxylic acid; $\alpha-K A$-keto acid; $\alpha-A A \alpha$-amino acid; Glu- $\gamma-S A$ Glutamic- $\gamma$-semialdehyde

acid containing protein, functioning as an aminomethyl carrier. The result of the $\mathrm{P}$ and $\mathrm{H}$ protein action is the liberation of $\mathrm{CO}_{2}$. The T protein is a tetrahydrofolate requiring enzyme, a flavoprotein which is reduced in the presence of the $\mathrm{H}$ protein and glycine, transferring carbon 2 of glycine to tetrahydrofolate and liberating $\mathrm{NH}_{3}$. The $\mathrm{L}$ protein is a $\mathrm{NAD}^{+}$dependent lipoamide dehydrogenase, regenerating the $\mathrm{H}$ protein.

In the classic phenotype of nonketotic hyperglycinemia life-threatening illness develops in early postnatal life. Most patients exhibit listlessness, lack of spontaneous movement, opisthotonus, myoclonus and/or grand mal seizures, hiccups, and failure to thrive. Survivors develop chronic seizures and deep mental retardation. In the classic nonketotic hyperglycinemia a defective $\mathbf{P}$ or $\mathrm{T}$ protein has been designated. In atypical cases a defective $\mathrm{T}$ protein and in a patient with a progessive neurodegenerative disorder a defective $\mathrm{H}$ protein have been reported.

Characteristic for nonketotic hyperglycinemia is that glycine concentration is not only increased in plasma and urine, but also in other body fluids, especially in cerebrospinal fluid. Fig. 12 shows an amino acid chromatogram of urine in a case of nonketotic hyperglycinemia.

Apart from nonketotic hyperglycinemia, ketotic hyperglycinemia was described in the sixties. Ketotic hyperglycinemia is an associated finding in many (neonatal) organic acidurias. Secondarily hyperglycinuria occurs in:

1. Propionic acidemia

2. Methylmalonic acidemia 


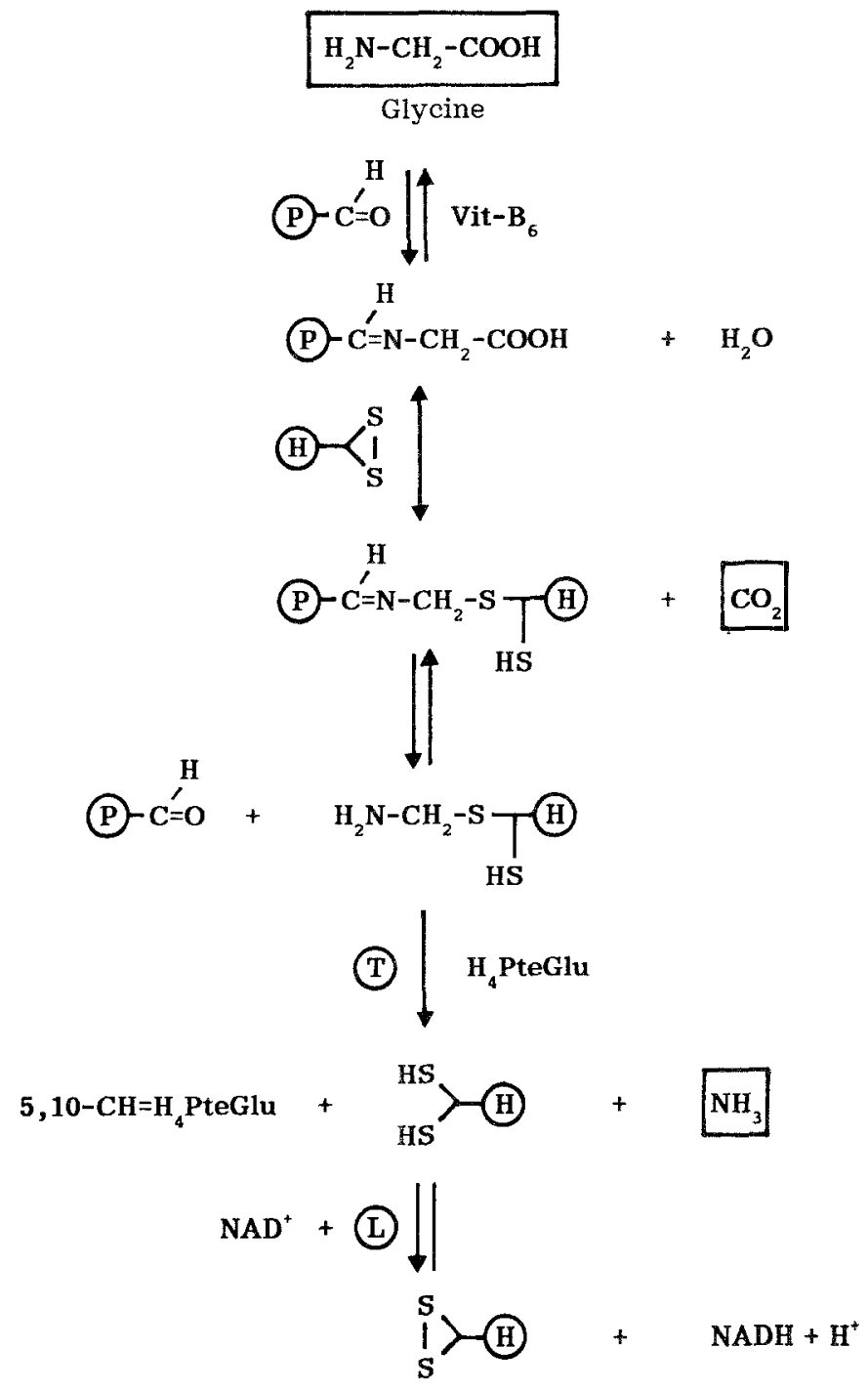

Fig. 11. Metabolic pathway of the glycine cleavage complex

3. Isovaleric acidemia

4. 2-Methylacetoacetic aciduria

5. D-Glyceric aciduria

6. Hyperprolinemia Type I and II

6. Iminoglycinuria

7. Infants up to 6 months of age

8. Starvation

9. Bacterial decomposition of hippuric acid

10. Valproic acid therapy

Organic acid analysis is therefore essential in the differential diagnosis of hyperglycinuria. In Fig. 13 an example of a chromatogram of amino acids in urine from a patient with propionic acidemia is shown. Propionyl-CoA is normally converted into D-methylmalonyl-CoA by propionyl-CoA carboxylase 


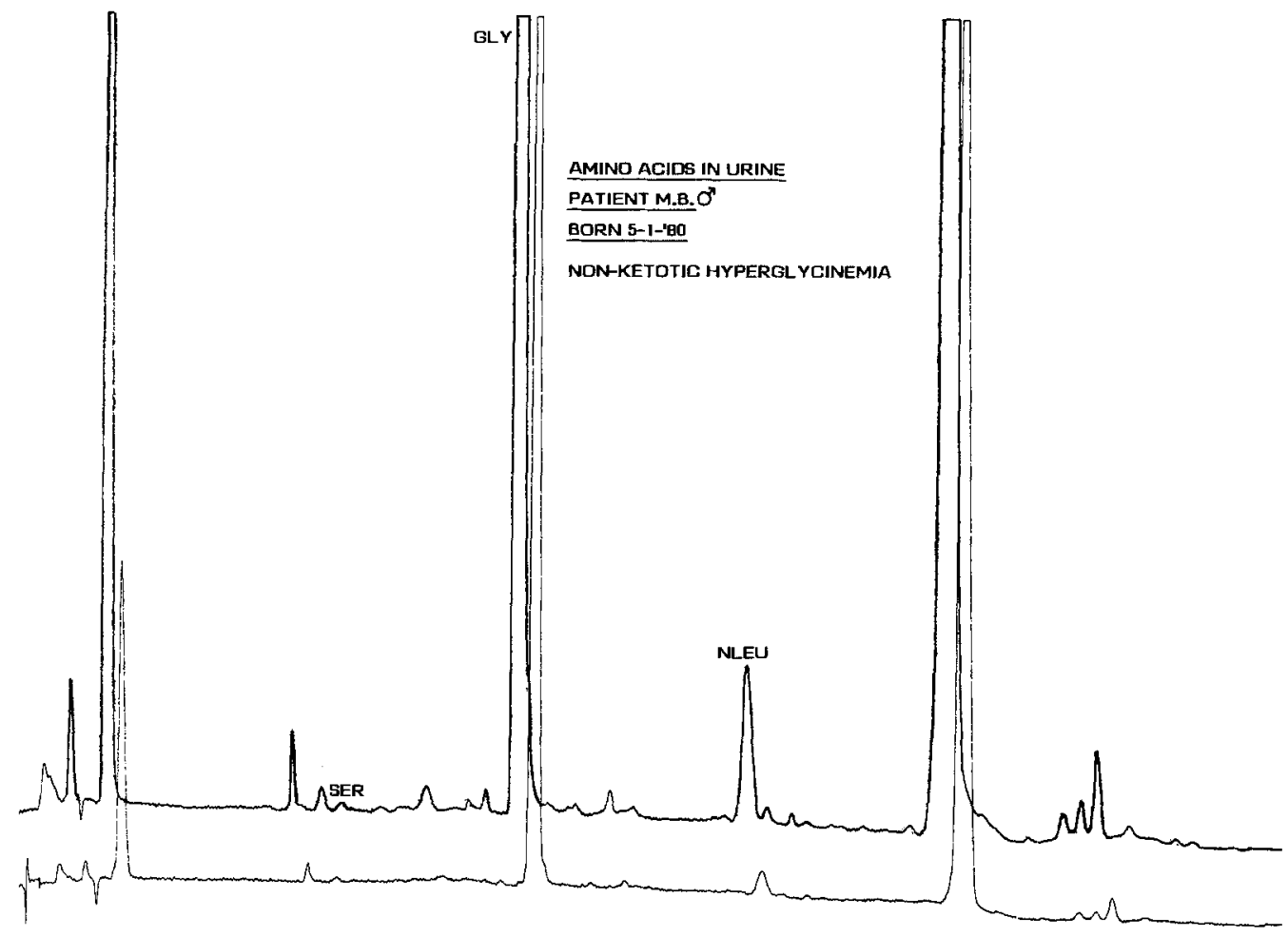

Fig. 12. Chromatogram of amino acids in urine from a patient with a defect in the glycine cleavage complex

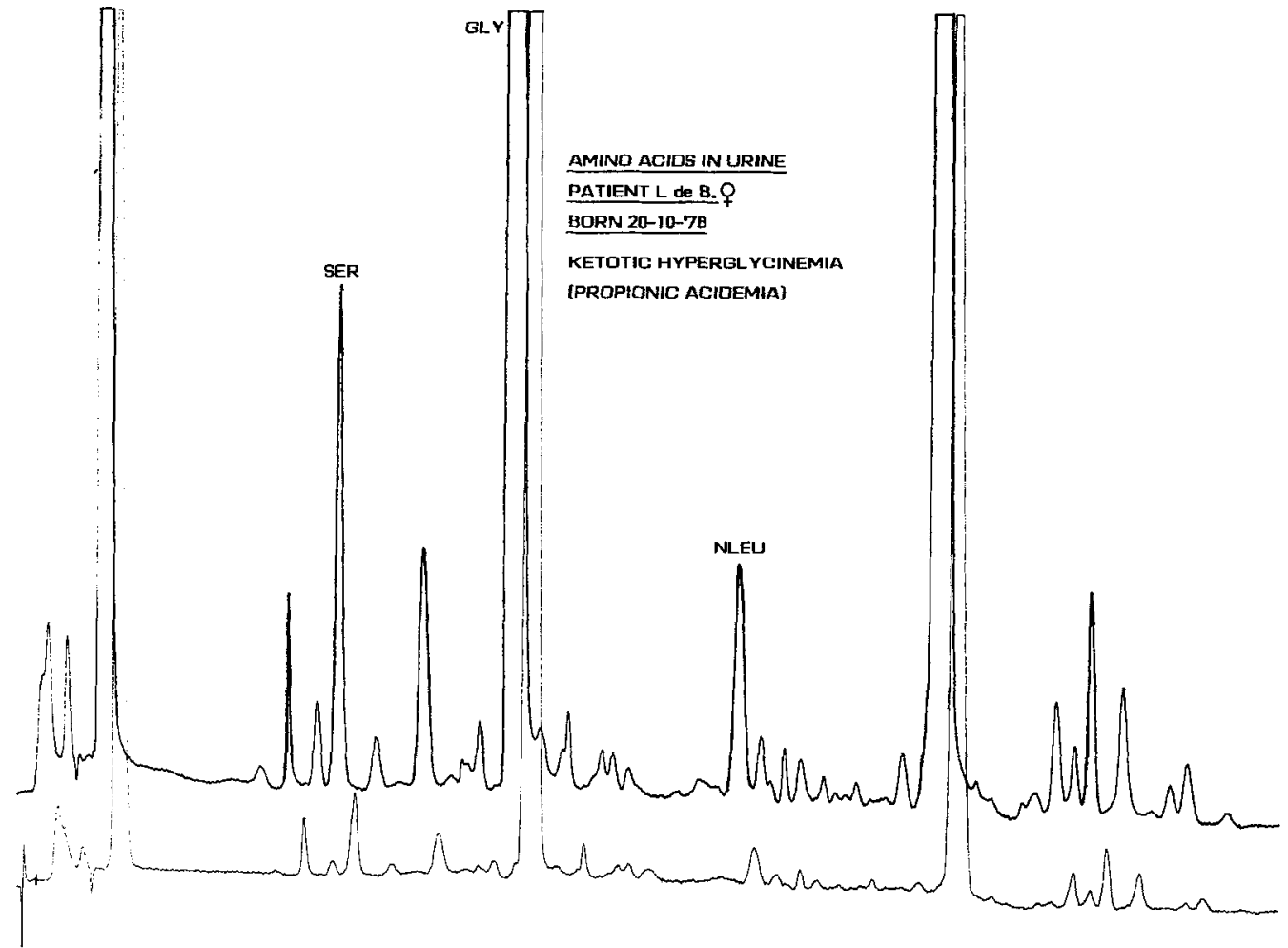

Fig. 13. Chromatogram of amino acids in urine from a patient with ketotic hyperglycinemia due to propionyl carboxylase deficiency 
(see Fig. 14). In propionic acidemia the mitochondrial biotine enzyme propionylCoA carboxylase is deficient. (McKusick 23200, 23205 and 25326). There are genetically distinct forms of propionic acidemia: an apoenzyme deficiency, and multiple carboxylase deficiencies (holocarboxylase synthetase deficiency and the late onset biotinidase deficiency). In all types of propionic acidemia biotine dependency might occur.

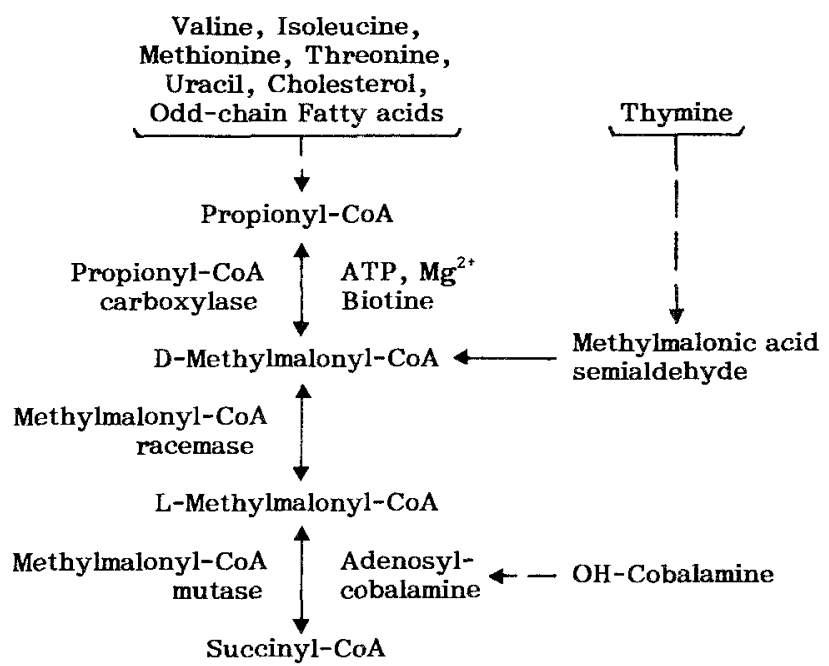

Fig. 14. Metabolic pathway of propionyl-CoA and methylmalonyl-CoA degradation

Hyperglycinuria e.g. also occurs in methylmalonic acidemia. (McKusick $25100,25110,25111,25112$, and 27741). In methylmalonic acidemia the conversion of D-methylmalonyl-CoA into succinyl-CoA is impaired, due to a deficiency of the apoenzymes methylmalonyl racemase or mutase, or to a defect in the cofactor adenosylcobalamine synthesis (see Fig. 14). Only in vitamin B12 dependency the prognosis of the disease is reasonable. In Fig. 15 the gas chromatogram of organic acids in urine from a patient with a vitamin B12 unresponsive methylmalonic acidemia is given. Besides a huge peak of methylmalonic acid, many peaks of propionic acid metabolites are also present. This is typical for the vitamin B12 unsensitive methylmalonic acidemia.

Glycine concentration in plasma and urine can be decreased in folic acid deficiency.

\section{Alanine}

No primary disorder of alanine metabolism is described. Urinary excretion of alanine is elevated in all disorders of lactic acidemia, and together with glutamine in all hyperammonemia syndromes (see also Fig. 17). Bacterial contamination of a urine can lead to an increase of alanine.

Alanine excretion in the urine is decreased in ketotic hypoglycemia and in disregulated patients with fatty acid $\beta$-oxidation disorders. 


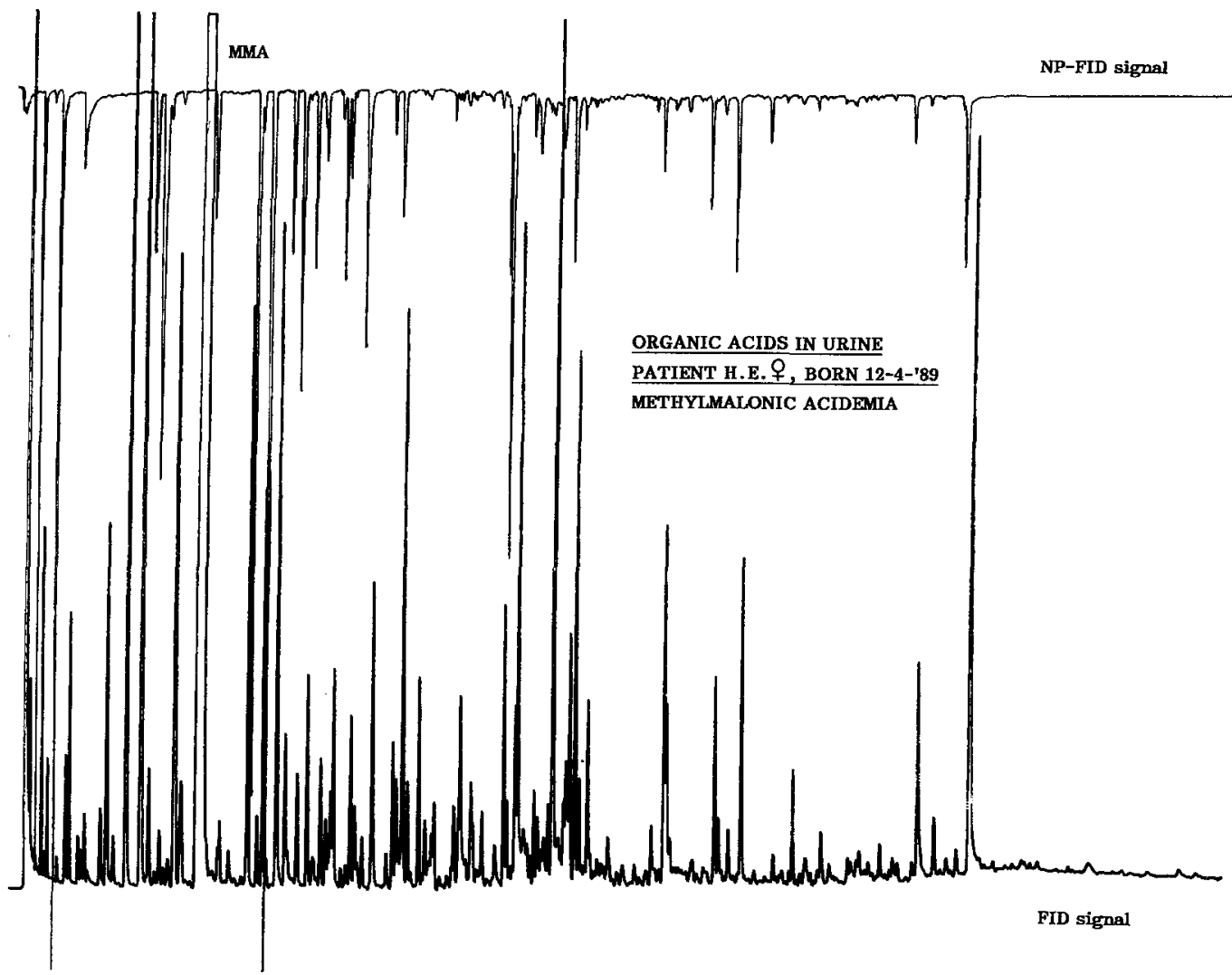

Fig. 15. Gas chromatogram of organic acids in urine from a patient with vitamin $\mathbf{B}_{12}$ unresponsive methylmalonic acidemia

\section{Hyperammonemia syndromes}

Disorders of urea synthesis usually cause hyperammonemia. Hyperammonemia can primarily arise from inborn errors in urea synthesis, or secondarily from inborn errors in the metabolism of ornithine, lysine, tyrosine, branched chain amino acid (organic acidemias), and from severe liver pathology.

The metabolic pathway of urea synthesis is shown in Fig. 16. The formation of carbamylphosphate from ammonia by action of mitochondrial carbamylphosphate synthetase (CPS) is the first step in urea synthesis. CPS needs $\mathrm{N}$ acetylglutamic acid as an allosteric activator. $\mathrm{N}$-acetylglutamic acid is synthesized by $\mathrm{N}$-acetylglutamate synthetase (NAGS). Arginine is an activator of NAGS, providing a mechanism for arginine to produce a positive feedback on ureagenesis. Mitochondrial ornithine transcarbamylase (OTC) transfers the carbamyl group from carbamylphosphate to ornithine under formation of citrulline. Citrulline leaves the mitochondria and reacts with aspartate to form argininosuccinic acid, activated by cytosolic argininosuccinic acid synthetase (AS). Argininosuccinic acid is cleaved by cytosolic argininosuccinase (AL) in fumaric acid and arginine. Finally, urea and ornithine are formed from arginine by action of cytosolic arginase. Ornithine enters the mitochondria, and the so-called urea cycle is closed. 


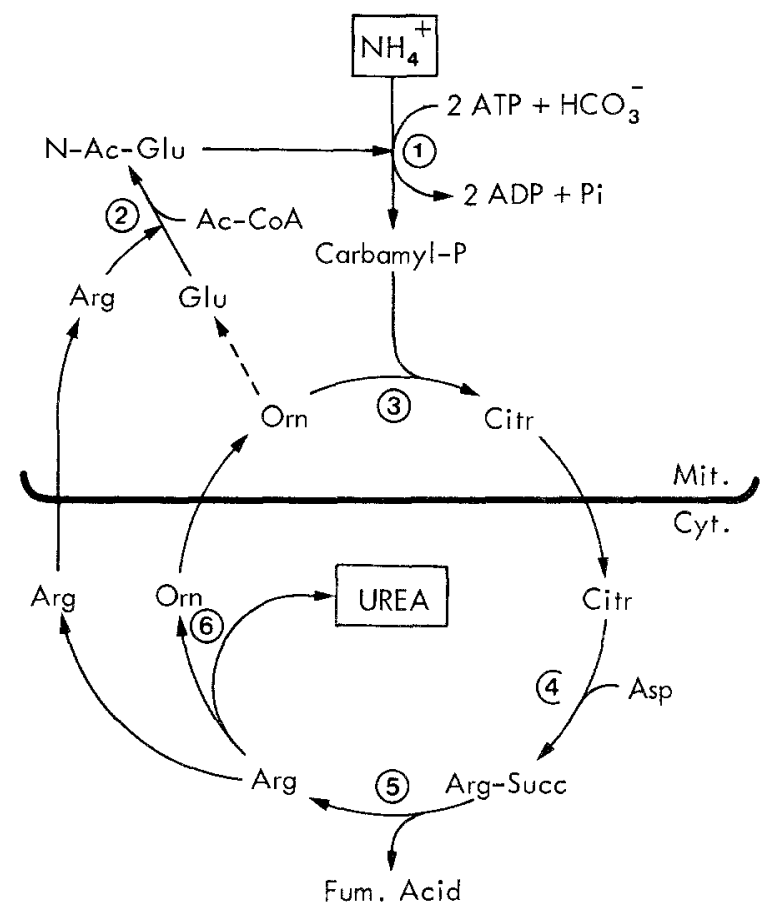

Fig. 16. Metabolic pathway of urea synthesis

Two distinct carbamylphosphate synthetases are distinguished: a mitochondrial ammonia dependent carbamylphosphate synthetase (CPS I), and a cytosolic glutamine dependent carbamylphosphate synthetase (CPS II), not activated by $\mathrm{N}$-acetylglutamic acid. CPS II is involved in the pyrimidine synthesis with orotic acid as an important intermediate. If carbamylphosphate accumulates, a high excretion of orotic acid in urine can be detected.

In the hyperammonemic state alanine, glutamine, and sometimes lysine are increased in plasma and urine of the affected patients (see Fig. 17). Ammonia induces glutamate dehydrogenase to form glutamic acid from $\alpha$-ketoglutaric acid, and induces glutamine synthetase to form glutamine from glutamic acid. Pyruvic acid is transaminated to alanine induced by glutamic acid. If $\alpha$-ketoglutaric acid is depleted, lysine accumulates by partial substrate depletion of lysine- $\alpha$-ketoglutarate reductase (see also Fig. 7).

The clinical presentation of neonatal hyperammonemia is mostly dramatic. Within 24 to 48 hours after birth the neonate may develop:

1. Nutritional problems as soon as protein is introduced

2. Progressive lethargy with hypothermia

3. Areflexia and alternately hypo- and hypertonia

4. Encephalopathy with brain edema and seizures

5. A sepsis-like picture, but with negative bacterial cultures and not reacting on antibiotic therapy

6. Coma

7. Sudden death 


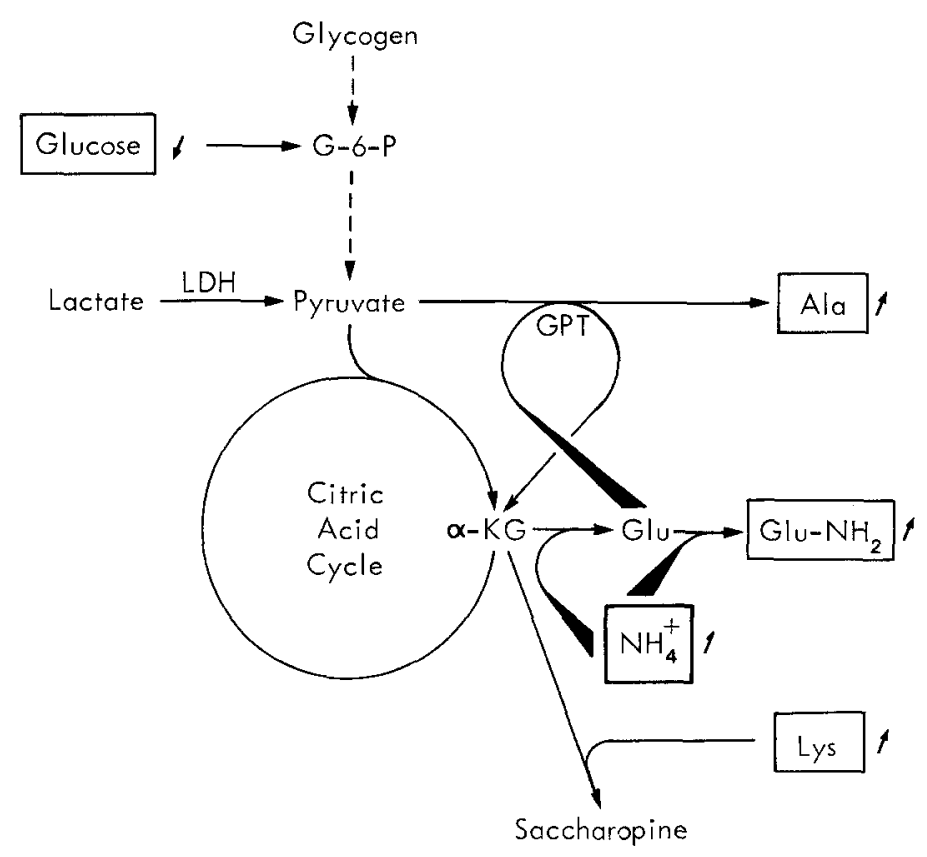

Fig. 17. Metabolic pathway explaining increased alanine, glutamine, and lysine production. $\uparrow$ increased; $\downarrow$ decreased

Some hyperammonemia syndromes may present later in infancy with episodic:

1. Protein aversion

2. Vomiting

3. Failure to thrive

4. Hair abnormalities (trichorrhexis nodosa)

5. Agitation and irritability

6. Cyclic migraine

7. Hepatomegaly

8. Hypo- and/or hypertonia

9. Stupor, coma and/or convulsions

For the differential diagnosis of primary hyperammonemia, see Table 1 . In CPS I (McKusick 23730) and NAGS (McKusick 23731) deficiency no abnormal excretion of urea cycle intermediates or orotic acid is observed. The only biochemical symptom of hyperammonemia can be an increase of alanine and glutamine excretion. As a consequence the diagnosis can only be confirmed by enzyme analysis in a liver biopsy. Some cases of CPS I deficiency present with the urinary organic acid excretion pattern as seen in 3-hydroxy-3-methylglutaric aciduria.

OTC deficiency (McKusick 31125) is an X-linked dominant inherited disease, which means that males are severly affected, whereas females are mildly affected or symptom-free. OTC deficiency can be differentiated from CPS or NAGS deficiency by the very high orotic acid excretion in the urine. Fig. 18 shows 
Table 1

\begin{tabular}{lcccccc}
\hline \multicolumn{7}{c}{ Amino acids } \\
\cline { 2 - 6 } $\begin{array}{l}\text { Enzyme } \\
\text { deficiency }\end{array}$ & $\begin{array}{c}\text { ALA }+ \\
\text { GLN }\end{array}$ & ORN & CIT & ASA & ARG & $\begin{array}{c}\text { Orotic } \\
\text { Acid }\end{array}$ \\
\hline CPS I & ++ & - & - & - & - & - \\
NAGS & ++ & - & - & - & - & - \\
OTC & +++ & - & - & - & - & +++ \\
AS & ++ & - & +++ & - & $\downarrow$ & ++ \\
AL & ++ & - & + & +++ & $\downarrow$ & + \\
Arginase & + & $\downarrow$ & - & - & +++ & ++ \\
\hline
\end{tabular}

+ increased; - normal; $\downarrow$ decreased

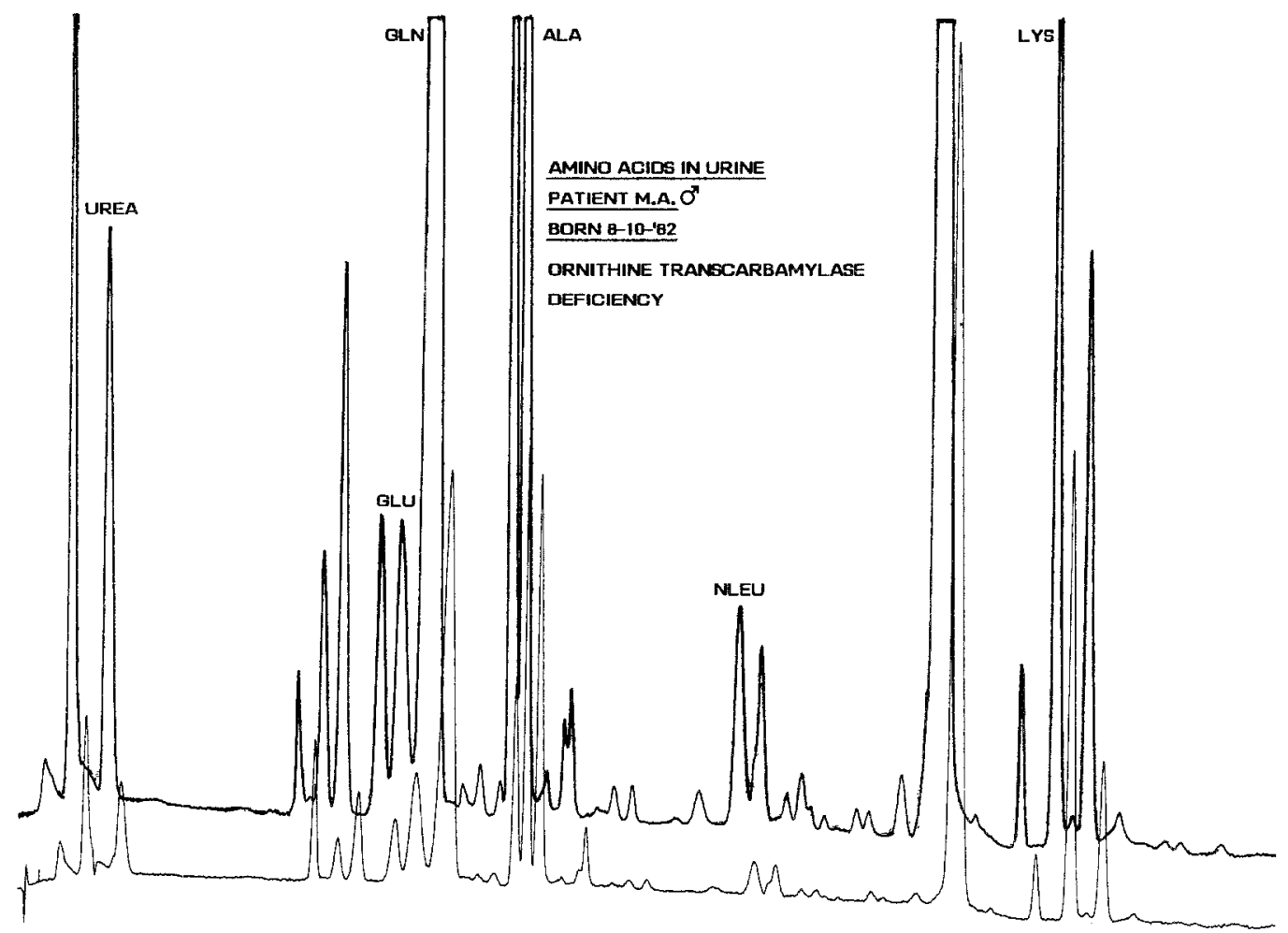

Fig. 18. Chromatogram of amino acids in urine from a patient with hyperammonemia due to ornithine transcarbamylase deficiency

the amino acid chromatogram of urine from a male patient with OTC deficiency. Note the very high excretion of GLN, ALA and LYS.

In AS deficiency (McKusick 21570) citrullinemia and citrullinuria with hypoargininemia will be observed. Orotic acid is mildly increased. Fig. 19 shows the amino acid excretion pattern in the urine from a treated patient with citrullinemia. Citrulline is already strongly elevated in the amniotic fluid from mothers with a fetus affected by citrullinemia [12]. 


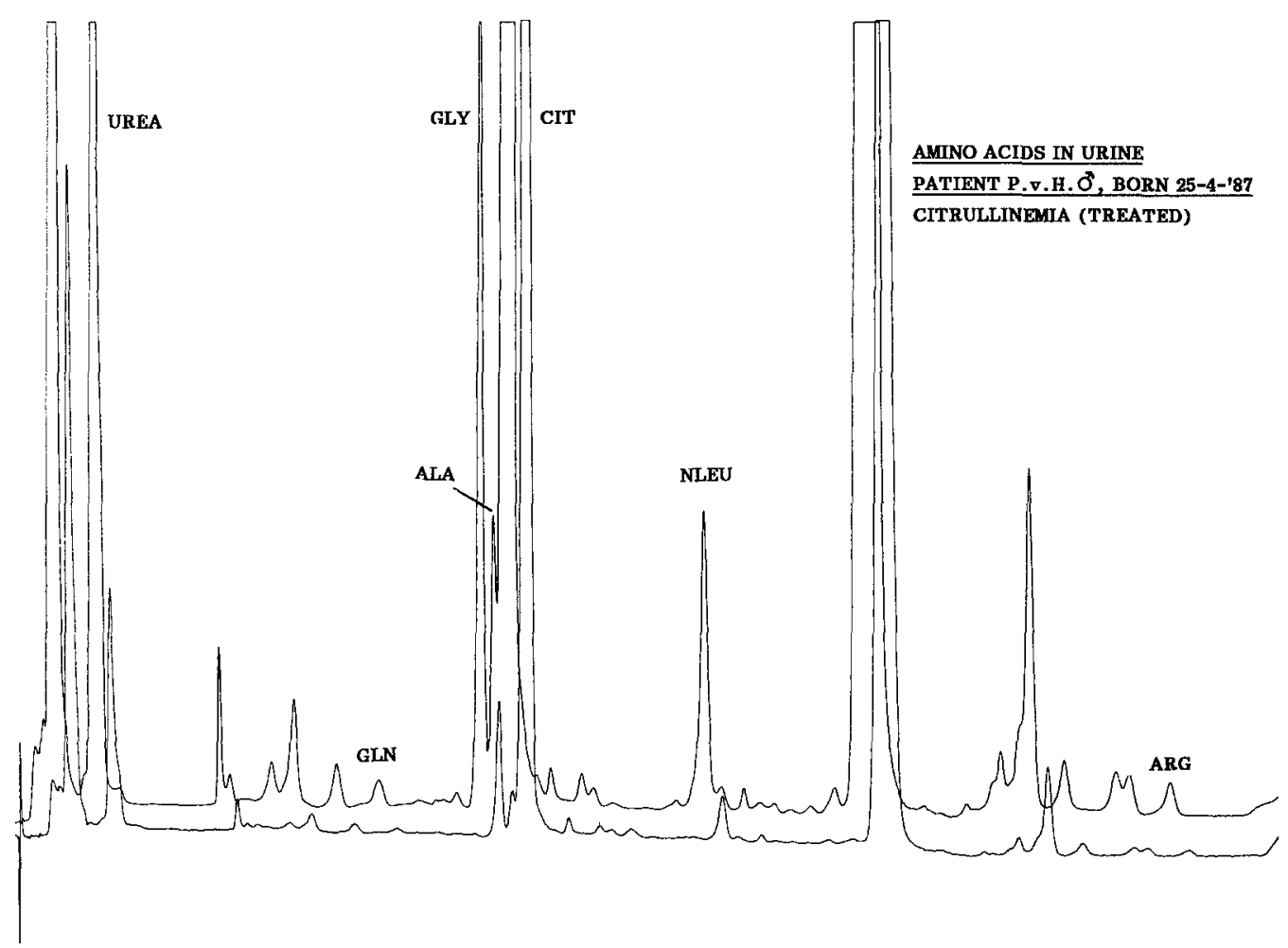

Fig. 19. Chromatogram of amino acids in urine from a patient with hyperammonemia due to argininosuccinic acid synthetase deficiency

In AL deficiency (McKusick 20790) a huge excretion of argininosuccinic acid and two cyclic metabolites can be observed (see Fig. 20). Citrulline is mildly and orotic acid slightly increased. If the fetus is affected, argininosuccinic acid is already increased in the amniotic fluid [13]. During the chromatographic run the cyclic metabolites are spontaneously formed, which explains the baseline shift behind the argininosuccinic acid peak. Arginosuccinic acid elutes at the same time as the internal standard NLEU.

In arginase deficiency (McKusick 20780) hyperargininemia and hyperargininuria occur. The urine may contain increased concentrations of cystine, ornithine and lysine. Urinary orotic acid excretion is massive. The hyperammonemia might be mild. Patients with argininemia excrete monosubstituted guanidino compounds [14].

Secondary hyperammonemia can occur in:

1. Tyrosinemia type I

2. Hepatitis or severe liver pathology

3. Reye's syndrome

4. Lysinuric Protein Intolerance Syndrome

5. Organic acidemias

For a long time, primary hyperammonemia could hardly be treated. Starting in the early eithies new possibilities became available using the concept of 


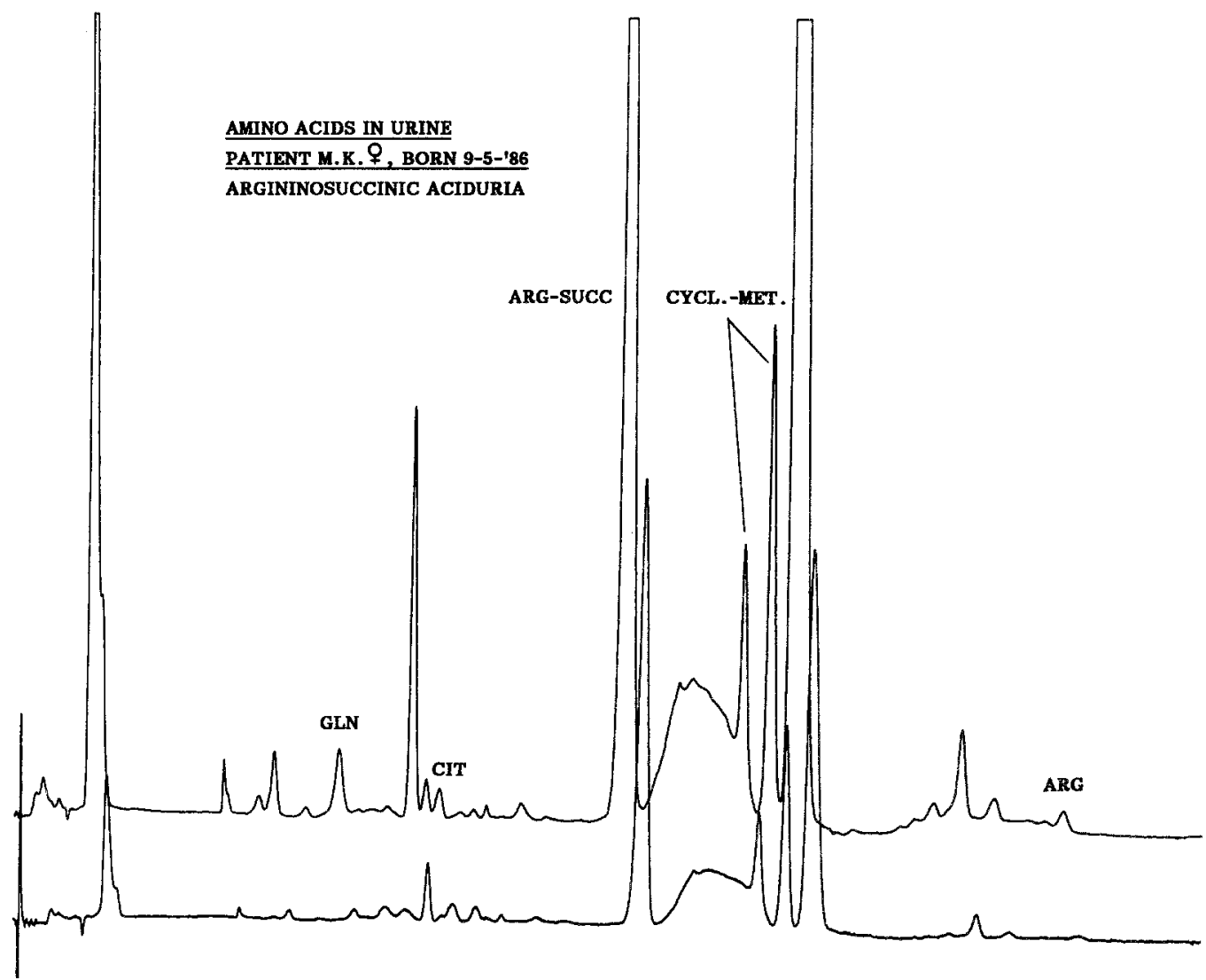

Fig. 20. Chromatogram of amino acids in urine from a patient with hyperammonemia due to arginosuccinase deficiency. The urine is diluted $5 \mathrm{x}$

alternative pathways of waste-nitrogen excretion in urine. For a overview see chapter 20 in [6].

\section{Citrulline}

The primary inborn error of citrulline metabolism is described above under 15. hyperammonemia syndromes. Citrulline excretion in urine can secondarily be increased in the two other urea cycle disorders argininosuccinic aciduria and argininemia, in saccharopinuria, and in renal insufficiency.

\section{7. $\alpha$-Aminobutyric acid}

There is no primary metabolic disorder of $\alpha$-aminobutyric acid metabolism described. An increased urinary $\alpha$-aminobutyric acid excretion can be observed in general aminoaciduria.

\section{Valine}

Only one patient is described with isolated hypervalinemia due to valine transaminase deficiency, in a child with vomiting, failure to thrive, and drowsi- 
ness [15]. (McKusick 27710). See enzymatic step 1 in Fig. 23. The parents showed also hypervalinuria.

Valine is also increased in maple syrup urine disease (see 23. branched chain amino acids).

\section{Cystine}

No disorders of cysteine or cystine metabolism are described. There are only transport disorders. In early-onset or infantile nephropathic cystinosis (McKusick 21980) free, nonprotein cystine accumulates within the lysosomes up to levels of $1000 \times$ normal. A defect in the ATP-dependent lysosomal efflux system has been detected. Cystine storage is observed in most tissues, which leads to tissue damage. The diagnosis can be made by cystine analysis in leukocytes or cultured fibroblasts. In patients older than 1 year of age corneal cystine crystals can be observed using slitlamp examination. In the second half year of life children with cystinosis develop a renal tubular Fanconi syndrome, including dehydration, acidosis, vomiting, electrolyte imbalances, hypophosphatemic rickets and growth retardation.

There is also a late-onset or adolescent nephropathic type of cystinosis (McKusick 21990). In this form nephropathy manifests itself early in the second decade of life, with proteinuria due to glomerular damage rather than tubular damage as seen in the early-onset form. Cystine storage in white cells is in the heterozygote range of the early-onset type of cystinosis.

Another cystine transport disorder is cystinuria (McKusick 22010). Of all aminoacidurias, cystinuria is the most frequent one. By mass screening it was discovered that one in every $2,000-15,000$ individuals suffers from cystinuria. Most patients with cystinuria present themselves with urinary-tract infections and urolithiasis.

There are two transport systems for cystine, one with a high affinity and low $K_{m}$, which is shared with the dibasic amino acids ornithine, lysine and arginine, and another with a low affinity and high $K_{m}$, not shared with the dibasic amino acids. Isolated cystinuria without dibasic amino aciduria is only described in one patient [16]. We discovered another case, from which the amino acid chromatogram in urine is shown in Fig. 21. In these patients a defect in the low affinity, high $K_{m}$ unshared renal transport system for cystine is considered.

Classical cystinuria with dibasic aminoaciduria is heterogeneous. Three types of cystinuric patients can be distinguished on the basis of intestinal biopsy investigations. In type $I$, there is no accumulation of cystine and the dibasic amino acids against a gradient. On an oral loading test with cystine, no increase in cystine can be detected in plasma. In type II, cystine accumulates activily, but the dibasic amino acids do not. The oral cystine loading test has the same result as in type I. In type III, cystine and the dibasic amino acids accumulate, but in a diminished fashion. A cystine tolerance test will be normal. Heterozygotes for type II and III can be detected by an increased excretion of mainly cystine and lysine. 


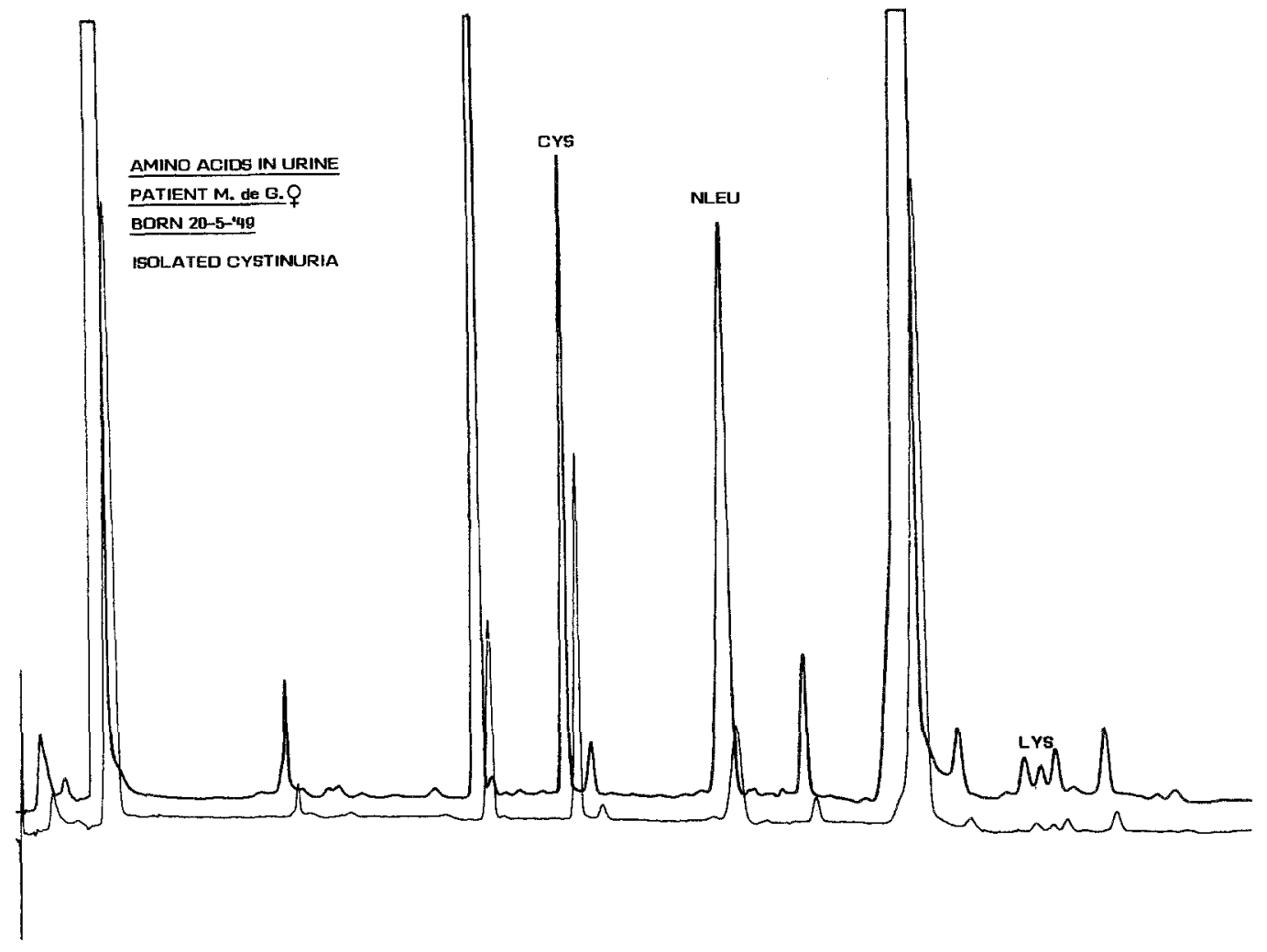

Fig. 21. Chromatogram of amino acids in urine from a patient with an isolated cystine transport defect

Fig. 22 shows the amino acid chromatogram of urine from a patient with classical cystinuria. Cystine and the dibasic amino acids ornithine, lysine and arginine are largely increased. In this patient the mixed disulfide cysteinylhomocysteine is clearly detectable.

Cystine excretion is also mildly increased in homocystinuria due to a remethylation defect (see Fig. 33), and in aminoacidopathies affecting the dibasic amino acids. In young infants during the first months of life, and in renal diseases cystine and lysine excretion can be increased.

\section{Homocitrulline}

No primary disorder of homocitrulline metabolism is described. Homocitrulline is secondarily increased in young infants up to one year of life, and in various hyperammonemia syndromes (see $\mathrm{HHH}$-syndrome at 33. Ornithine).

\section{Methionine}

A primary disorder of methionine metabolism is hypermethioninemia due to methionine adenosyltransferase, by which S-adenosylmethionine biosynthesis is impaired. (McKusick 25085) (see Fig. 31). On the basis of enzyme studies it was concluded, that in hypermethioninemia patients a high $K_{m}$ form of hepatic 


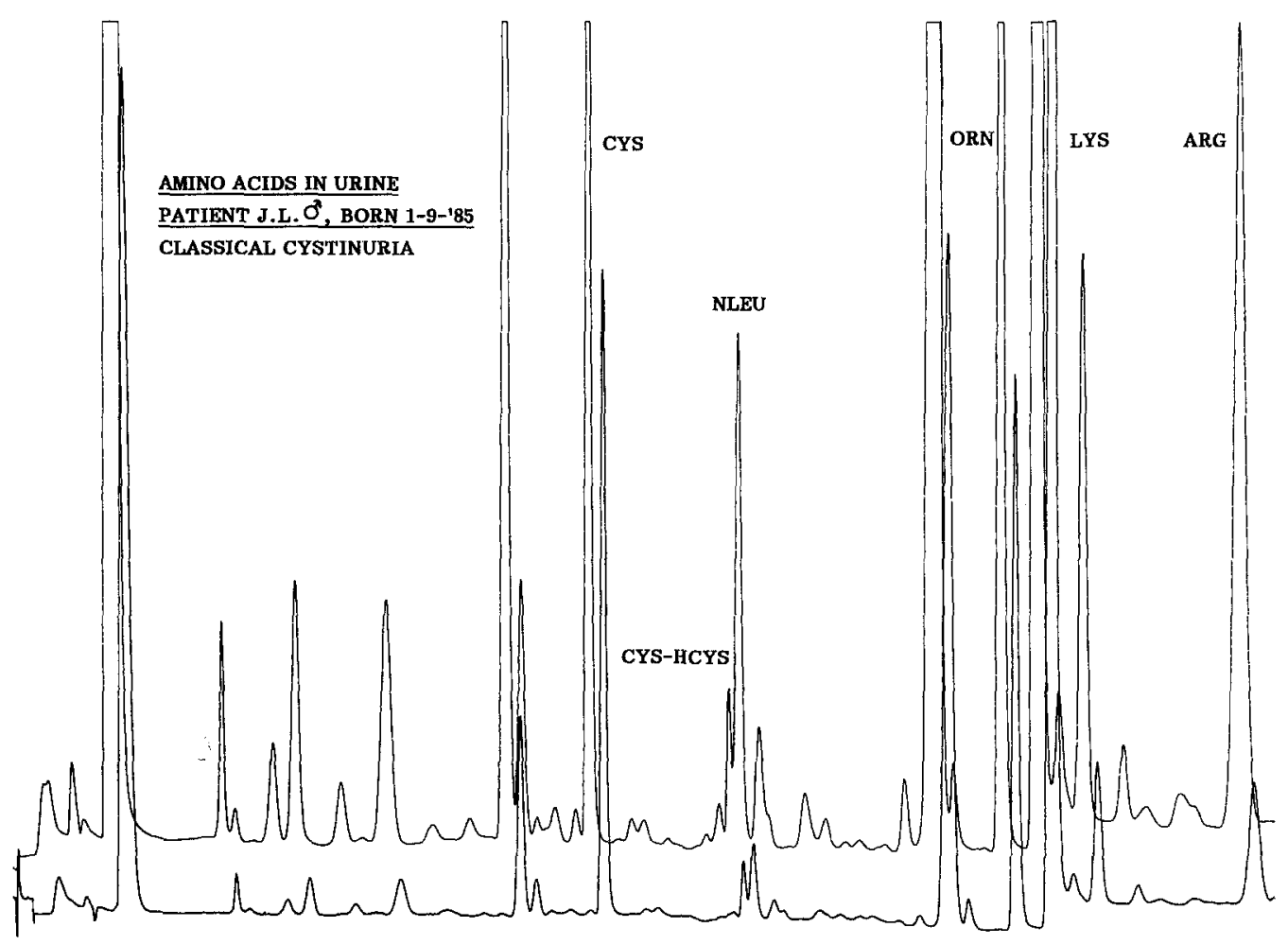

Fig. 22. Chromatogram of amino acids in urine from a patient with a transport defect of cystine and the dibasic amino acids

methionine adenosyltransferase is deficient, whereas the low $K_{m}$ form is unaffected. Besides increased methionine concentrations in plasma and urine, urinary elevations of methionine sulfoxide and 4-methylthio-2-oxobutyrate are detectable. In one adult patient dimethylsulfide was present in his breath.

Most patients were discovered in the neonatal period by mass screening for homocystinuria. Clinically, patients with a deficiency of the high $K_{m}$ form of methionine adenosyltransferase deficiency are virtually normal.

Hypermethioninemia and -uria can secondarily occur in homocystinuria (cystathionine- $\beta$-synthase deficiency), in severe generalized liver disease, in association with tyrosinemia type I, and with elevated serum folate. In neonates a transient hypermethioninemia has been reported. In patients with a remethylation defect of homocysteine plasma methionine can be decreased.

Two patients have been documented with a presumed methionine malabsorption syndrome (Oasthouse disease) (McKusick 25090). The patients were excreting high amounts of $\alpha$-hydroxybutyric acid, causing the abnormal smell of the patients. The patients had diarrhea, hyperpnea, convulsions, strikingly white hair and blue eyes, and were mentally retarded.

\section{Cystathionine}

Cystathionine is an intermediate metabolite of homocysteine conversion into cysteine (see Fig. 31). Cystathioninuria occurs in $\gamma$-cystathionase deficiency. 
(McKusick 21950). Most of the cysthationinuria patients $(80 \%)$ have no abnormal clinical symptoms. A minor part of the patients $(20 \%)$ have mental retardation with a wide assortment of other clinical abnormalities.

Cystathionine can secondarily be increased in: premature infants, vitamin $\mathrm{B}_{6}$ deficiency, thyrotoxicosis, liver cirrhosis, neuroblastoma, ganglioblastoma, and hepatoblastoma.

\section{Branched chain amino acids}

Following uptake by the cell, the essential branched chain amino acids valine, isoleucine and leucine are either incorporated into proteins or catabolized for energy. The first catabolic step is a transamination to the branched chain ketoacids by aminotransferases with mostly a-ketoglutarate as the amino-group acceptor (see step 1 in Fig. 23). A deficiency of valine aminotransferase was already mentioned under 18. valine. A single report for a French family mentions a disorder in leucine and isoleucine transamination [17]. A brother and sister presented at 2 to 3 months of age with seizures, failure to thrive, and mental retardation. They showed hyperleucinemia and isoleucinemia. The urinary amino acid excretion was normal.

VALINE $\stackrel{(1)}{\longrightarrow} \alpha$-Ketoisovaleric acid $\stackrel{\text { (2) }}{\longrightarrow}$ Isobutyryl-CoA $--\rightarrow$
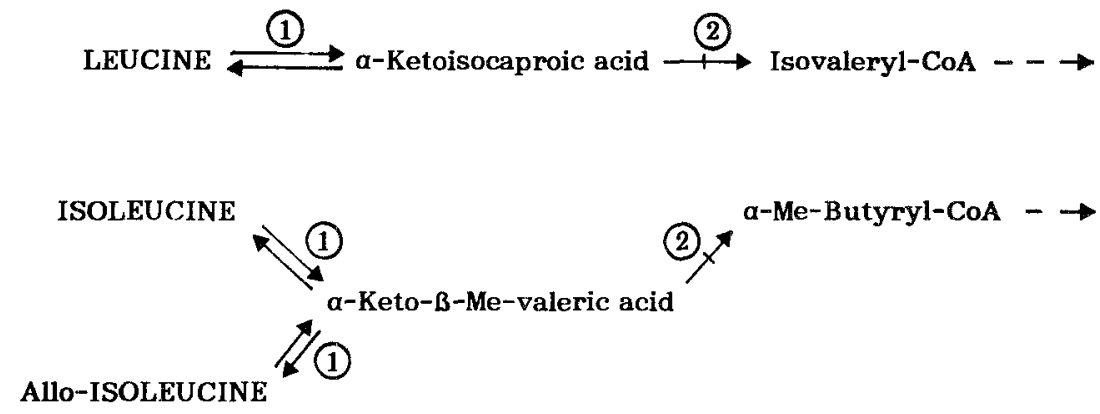

Fig. 23. Metabolic pathway of the first two steps in branched chain amino acid degradation

The second step in branched chain amino acid degradation is a decarboxylation and CoA-esterification of the branched chain $\alpha$-keto acids, by the branched chain ketoacid dehydrogenase complex (see step 2 in Fig. 23). The mitochondrial dehydrogenase complex consists of four major proteins:

1. A decarboxylase $E_{1}$ comprising two subunits $\alpha$ and $\beta$. The $E_{1} \alpha$ subunit binds thiaminepyrophosphate, creating the keto acid binding site for the release of $\mathrm{CO}_{2}$. The $E_{1} \beta$ subunit is thought that it functions in the transfer of the acyl moiety to $E_{2}$-lipoate.

2. An acyltransferase $E_{2}$ containing a covalently bound lipoate, catalyzing the transfer of the decarboxylated branched chain acyl moiety to CoA. 
3. Lipoamide dehydrogenase $E_{3}$, a flavoprotein, reoxidizing the reduced lipoate created by the $E_{2}$ reaction. The same protein also functions with pyruvate dehydrogenase (PDH) and $\alpha$-ketoglutarate dehydrogenase (KGDH) complexes.

Inherited protein defects in the branched chain $\alpha$-keto acid dehydrogenase complex lead to the metabolic disorder Maple Syrup Urine Disease (MSUD). There are six variants of MSUD: a classical form (neonatal to early childhood), an intermediate form (infant to adult), an intermittent form (childhood to adult), a thiamine responsive form (infant to adult), and forms with a specific deficiency of $E_{1} \beta, E_{2}$, or $E_{3}$ (all neonatal) (McKusick 24860).

The clinical picture varies from sudden apnea, ketoacidosis, coma, and death during the neonatal period to poor feeding, lethargy, (intermittent) ataxia, failure to thrive, and recurrent ketoacidosis during infancy. If untreated patients survive the first weeks of life, EEG abnormalities, severe psychomotor retardation, generalized dystonic posturing and other structural brain dysfunction are the rule.

Amino acid analysis gives very high levels of leucine and high levels of valine, isoleucine and allo-isoleucine in plasma and urine (see Fig. 24). In normal urine allo-isoleucine is not detectable, as a result of $\alpha$-keto- $\beta$-methylvaleric acid accumulation the rate of keto-enol tautomerization in this metabolite is sufficient to produce the stereoisomeer allo-isoleucine.

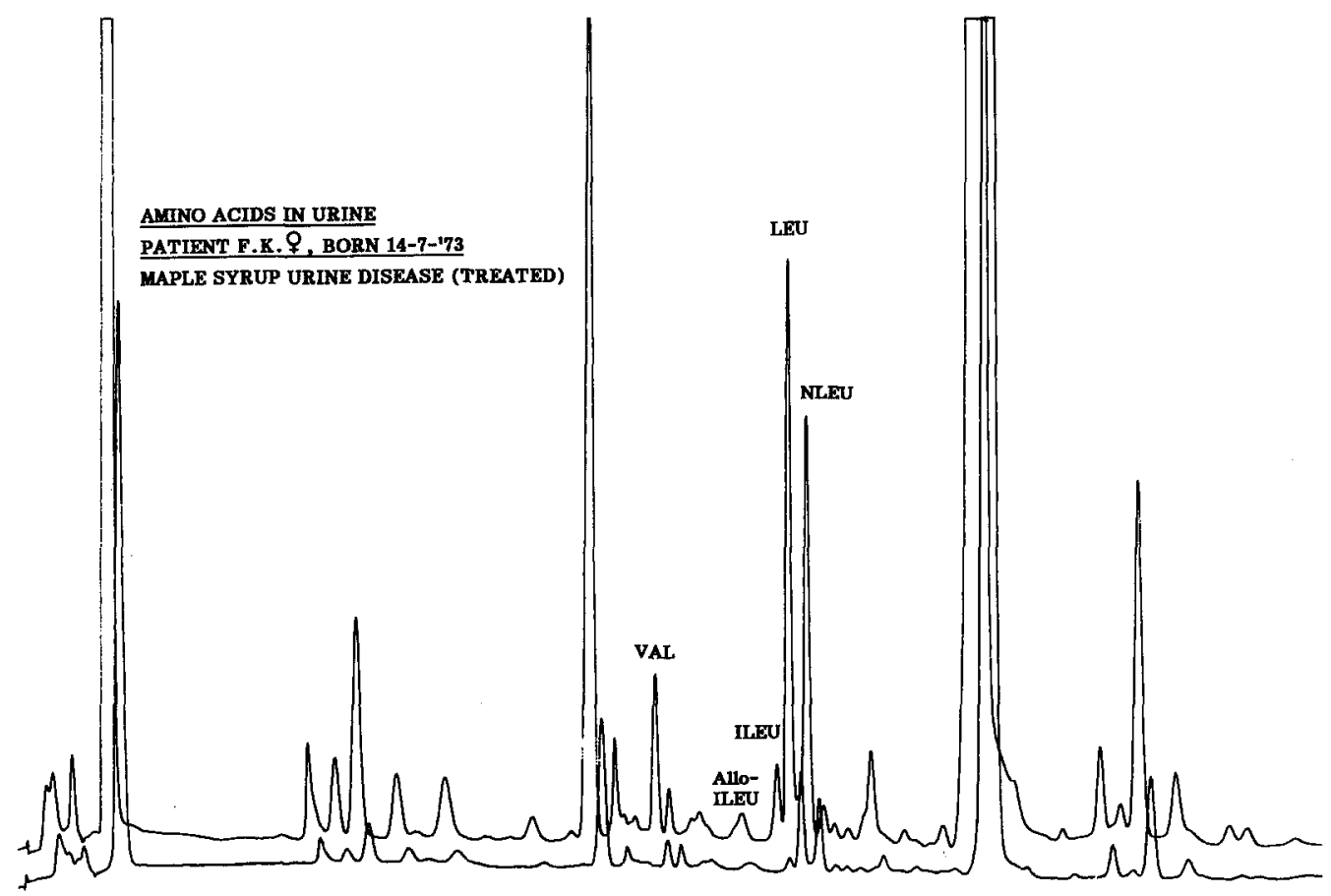

Fig. 24. Chromatogram of amino acids in plasma from a treated patient with maple syrup urine disease 
The primary diagnosis can be confirmed by gas chromatography of organic acids in urine. Huge peaks of $\alpha$-ketoisovaleric acid, $\alpha$-ketoisocaproic acid, and $\alpha$-keto- $\beta$-methylvaleric acid are detectable.

\section{Argininosuccinic acid}

The primary inborn error argininosuccinic aciduria is previously described under 15. hyperammonemia syndromes. Secondarily argininosuccinic acid might be detectable in hyperornithinemia.

\section{Tyrosine}

The metabolic pathway of tyrosine degradation is shown in Fig. 25. In a first reaction tyrosine is transaminated into 4-hydroxyphenylpyruvic acid. There are separate tyrosine aminotransferases in cytosol and mitochondria. The next step is the conversion of 4-hydroxyphenylpyruvic acid into homogentisic acid by the cytosolic enzyme 4-hydroxyphenylpyruvic acid dioxygenase. In a reaction of homogentisic acid oxidase, maleylacetoacetic acid is formed from homogentisic acid. Maleylacetoacetic acid is isomerized into fumarylacetoacetic acid, which is hydrolized by action of fumarylacetoacetate hydrolase (also called fumarylacetoacetase).

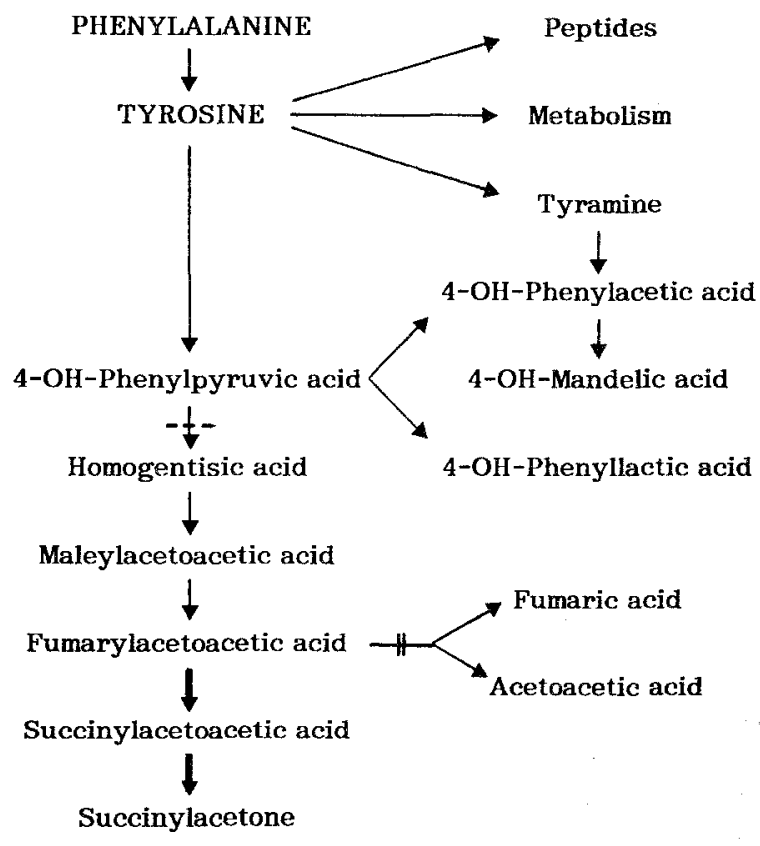

Fig. 25. Metabolic pathway of tyrosine degradation

In tyrosinemia type I (McKusick 27670) the enzyme fumarylacetoacetate hydrolase (FAH) is deficient. Within the same family an acute and chronic form 
can exist. The degree of residual FAH activity may determine whether the disease will be acute or chronic in the affected patient. In the acute form, there is failure to thrive, vomiting, diarrhea and a cabbagelike odor during the first week of life. In most patients hepatomegaly, fever, jaundice, ascites, edema, bleeding episodes, melena and epitaxis are seen to a varying degree. Untreated patients die within six to eight months. In the chronic form of the disease the clinical picture is similar but milder. The patients develop chronic liver disease, renal tubular dysfunction (deToni-Debre-Fanconi syndrome), and hypophosphatemia with rickets. Death occurs usually during the first decade. Hepatoma is a late complication in at least one third of the patients. Early liver transplantation is nowadays in most patients the ultimate therapeutic solution.

Amino acid analysis reveals tyrosinemia and methioninemia, and generalized amino aciduria with a prominent tyrosinuria (see Fig. 26). Organic acid analysis reveals tyrosyluria (4-OH-phenylpyruvic acid and 4-OH-phenyllactic acid) and succinylacetone and succinylacetoacetic acid. Due to FAH deficiency accumulating fumarylacetoacetic acid is reduced into succinylacetoacetic acid, which decarboxylates into succinylacetone (see Fig. 25). It is important to mention that succinylacetoacetic acid and succinylacetone can only be detected in fresh urine, because of rapid decomposition of these metabolites. Tyrosyluria originates from a partially reduced 4-OH-phenylpyruvic acid dioxygenase. Inhibition of $\delta$-aminolevulinic acid dehydratase by succinylacetone results in a high urinary excretion of $\delta$-aminolevulinic acid.

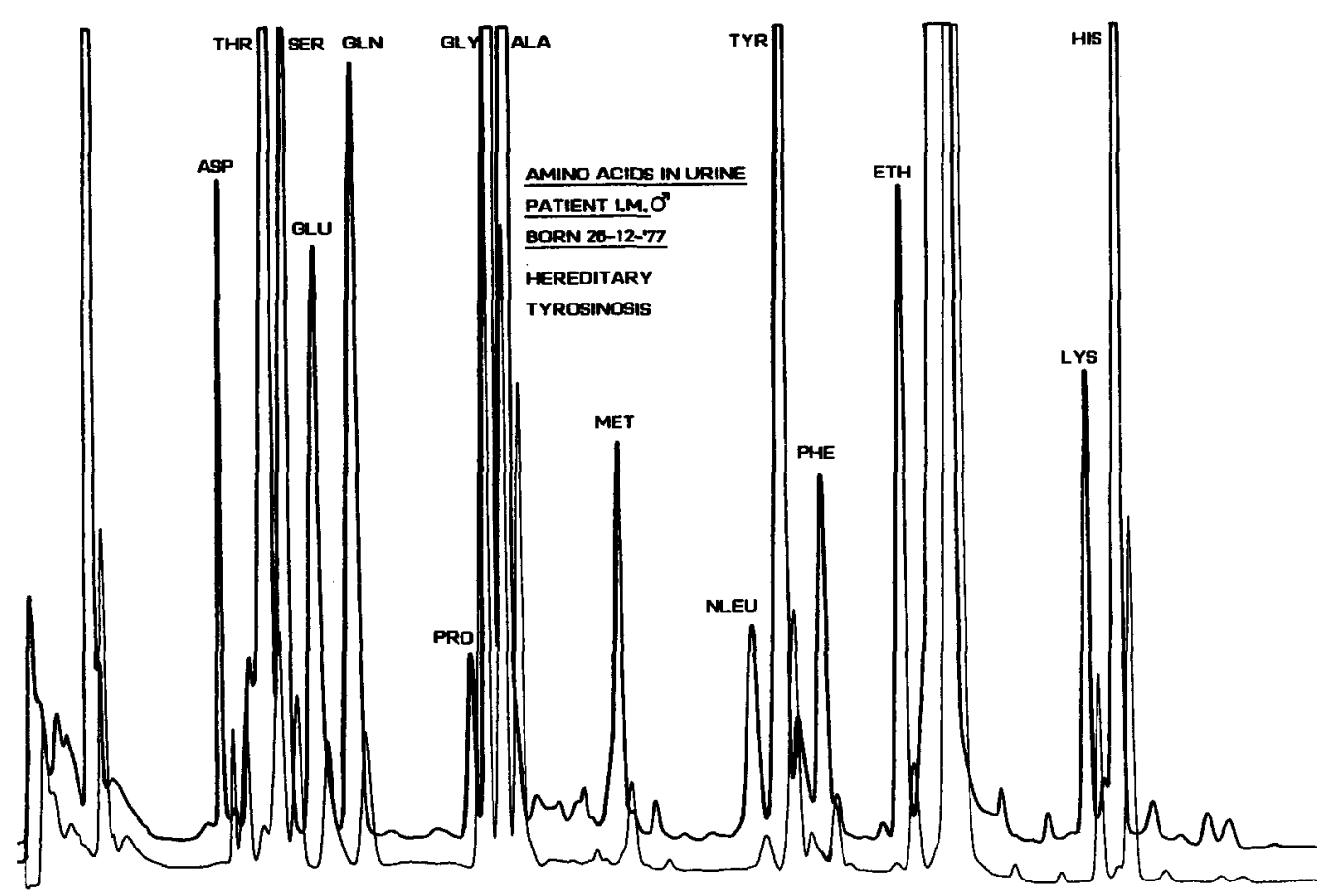

Fig. 26. Chromatogram of amino acids in urine from a patient with tyrosinemia type $I$ (fumarylacetoacetase deficiency) 
In tyrosinemia type II (Richner-Hanhart syndrome) cytosolic tyrosine transaminase is deficient. (McKusick 27660). The phenotype has also been described as: keratosis palmaris et plantaris. Originally Richner and Hanhart recognized a distinctive oculocutaneous syndrome. Clinically tyrosinemia type II is characterized by corneal erosions and plaques, palm and sole erosions, and hyperkeratosis usually occur during the first months of life, not responding to conventional therapy. Some patients develop mental retardation.

The striking abnormality in amino acid analysis is the tyrosinemia and tyrosinuria. In the gas chromatogram of organic acids only tyrosyluria can be observed, without the presence of succinylacetone and succinylacetoacetic acid.

In tyrosinemia type III 4-hydroxyphenylpyruvic acid dioxygenase is deficient. (McKusick 27671). For a long time it was considered that this enzyme was deficient in patients with tyrosinemia type I, but the enzyme activity was never lower than $20 \%$ of normal. Later FAH deficiency was discovered in tyrosinemia type I. The only patient described with tyrosinemia type III had normal FAH activity and no hepatic dysfunction. The clinical picture was that of mild mental retardation. Besides tyrosinemia and tyrosinuria, tyrosyluria was detected.

Neonatal tyrosinemia can secondarily occur in prematures and dysmatures, due to a high protein intake. Other possibilities for secondary tyrosinemia are galactosemia, congenital herpes infection, congenital lues, toxoplasmosis and

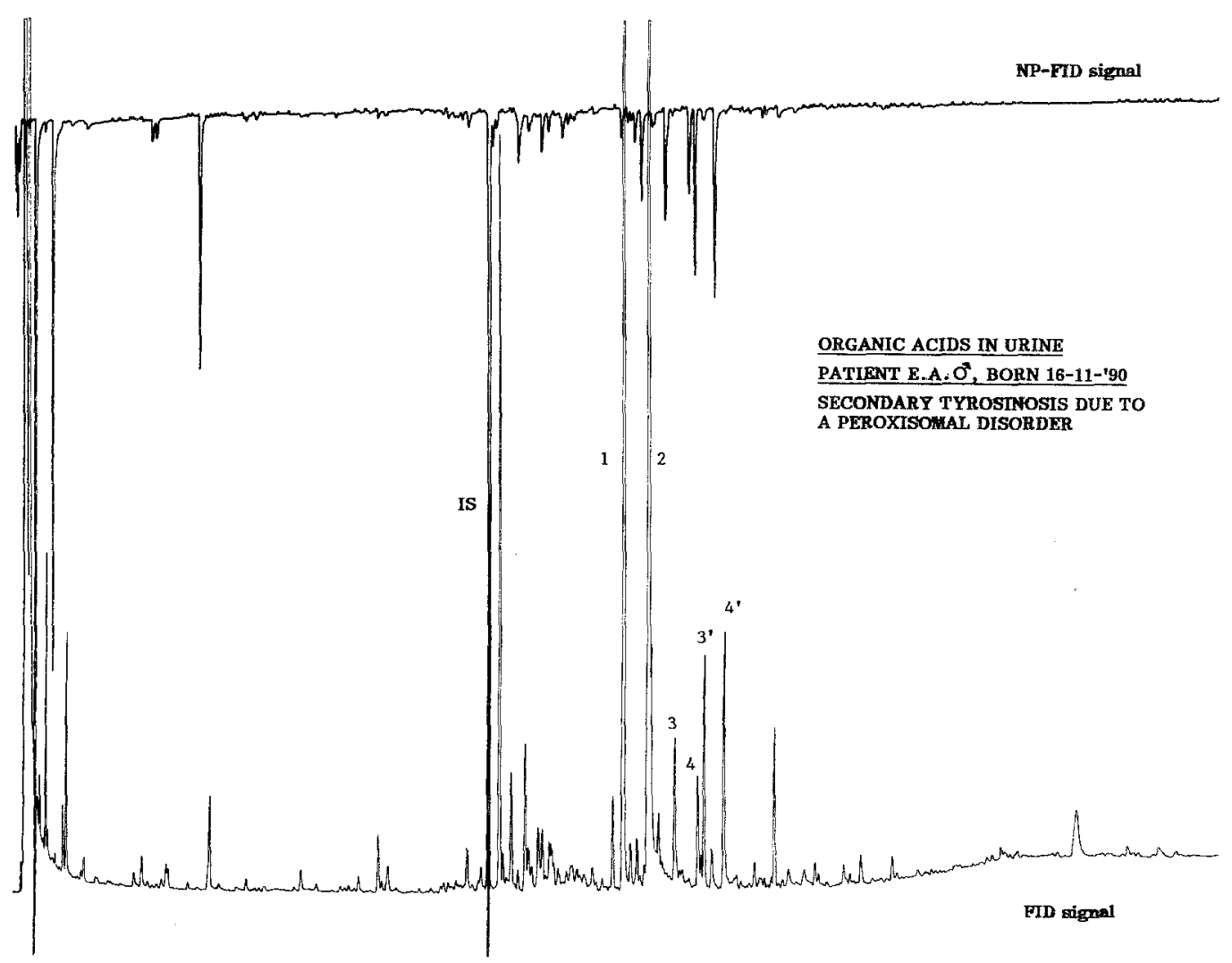

Fig. 27. Gas chromatogram of organic acids in urine from a patient with secondary tyrosinemia due to a peroxisomal disorder 
peroxisomal disorders (e.g. Zellweger syndrome) (see Fig. 27). A high excretion of tyrosine in the urine can be masked by antibiotics. In the amino acid chromatogram of urine a huge peak of an antibiotic metabolite is eluted at the same time as tyrosine (see Fig. 28). However the peak is broadened and other broad peaks with a high $440 \mathrm{~nm}$ signal are mostly present.

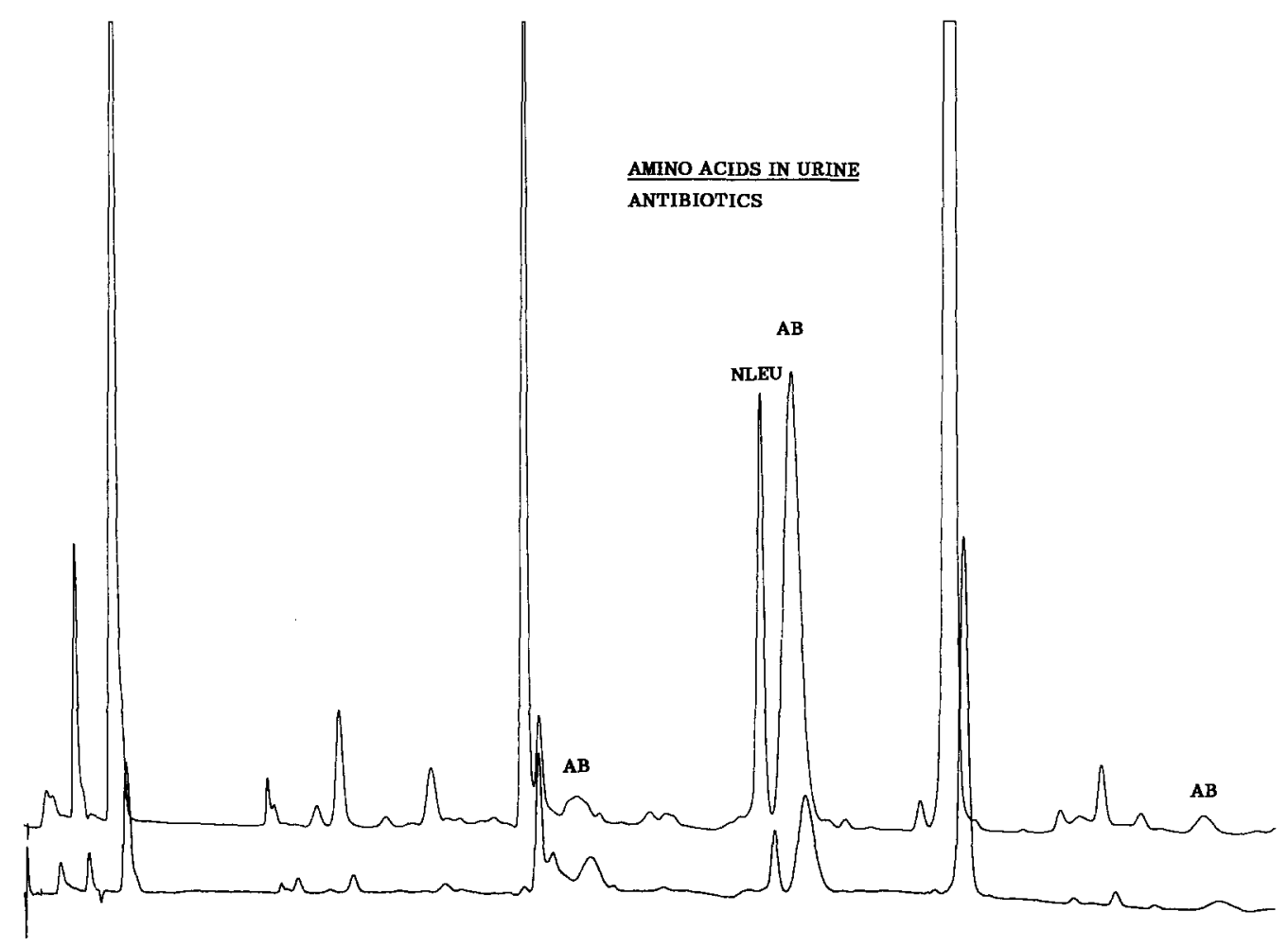

Fig. 28. Chromatogram of amino acids in urine from a patient treated with antibiotics

\section{6. $\beta$-Alanine}

In the sixties one single male infant has been described with a suggested deficiency of $\beta$-alanine- $\alpha$-ketoglutarate transaminase (McKusick 23740). The patient was convulsive. $\beta$-Alanine, $\beta$-aminoisobutyric acid and taurine were excreted in excess in the urine. Also $\gamma$-aminobutyric acid was detectable in urine.

More recently a patient has been reported with a large urinary excretion of 3 -hydroxypropionic acid, $\beta$-alanine, 3-hydroxyisobutyric acid, and $\beta$-aminoisobutyric acid [18], strongly suggesting a deficiency of methylmalonic semialdehyde and malonic semialdehyde dehydrogenase. The patient was discovered by newborn screening for hypermethioninemia. In the first year the patient had episodes of diarrhea and vomiting. The psychomotor development the patient had episodes of diarrhea and vomiting. The psychomotor development was normal.

$\beta$-Alanine excretion in urine is secondarily increased after kidney transplantation, when the kidney is rejected, and in carnosinuria. 


\section{Phenylalanine}

Direct or indirect disorders of phenylalanine metabolism cause hyperphenylalaninemia, generally known as phenylketonuria (PKU). PKU is one of the most studied inborn error of metabolism. Phenylalanine, an essential amino acid, is normally hydroxylated to tyrosine (step 1 in Fig. 29) by the enzyme phenylalanine hydroxylase (PAH). Tetrahydrobiopterin $\left(\mathrm{BH}_{4}\right)$ is needed as a cofactor to reduce molecular oxygen. Providing electrons, $\mathrm{BH}_{4}$ is oxidized to quinonoid-dihydrobiopterin $\left(\mathrm{qBH}_{2}\right) . \mathrm{BH}_{4}$ is regenerated from $\mathrm{qBH}_{2}$ by the enzyme dihydropteridine reductase (DHPR) (step 2 in Fig. 29). $\mathrm{BH}_{4}$ is de novo synthesized from guanosine triphosphate in several enzymatic steps.

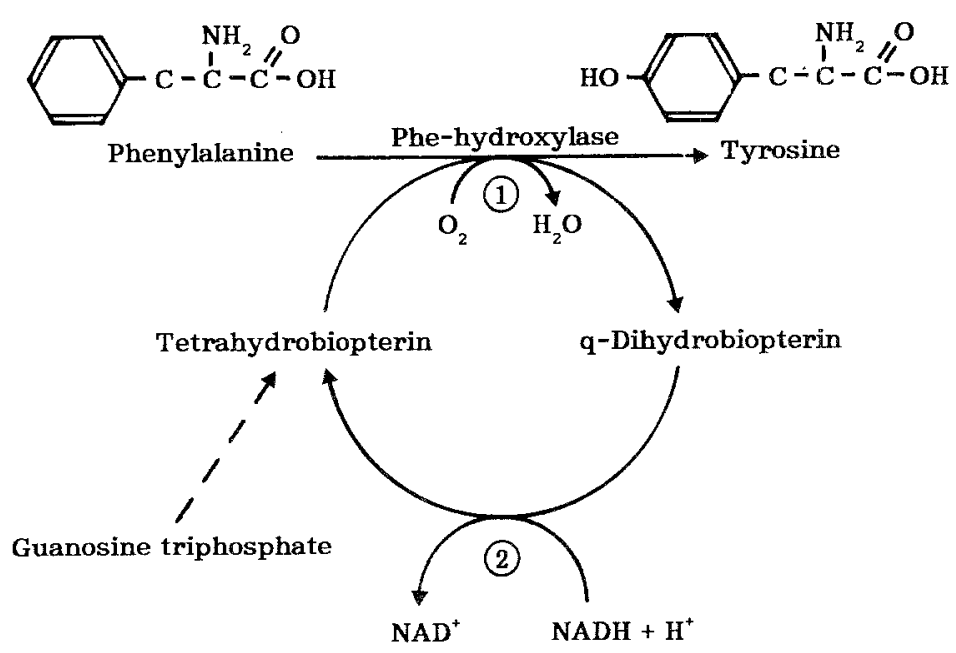

Fig. 29. Metabolic pathway of phenylalanine conversion into tyrosine

Classical hyperphenylalaninemia (PKU I) is caused by a deficiency of PAH. (McKusick 26160) (see Fig. 30). PAH activity is less than $2 \%$ of normal. Besides phenylalanine high urinary excretion of phenylpyruvic acid, phenyllactic acid, phenylacetic acid, mandelic acid and phenylglyoxylic acid can be detected by organic acid analysis. PKU is clinically expressed in mental retardation, behavioral abnormalities, dermatological abnormalities, hypopigmentation, and impaired physical growth affecting head circumference and height. The clinical symptoms of PKU are now of mainly historical interest, because the disease is prevented by neonatal screening.

There is also a variant form namely non-PKU hyperphenylalaninemia, in which PAH deficiency is less severe. If the phenylalanine concentration in plasma is less than $0.5 \mathrm{mmol} / 1$ no treatment is necessary.

Other types of PKU are the result of an impaired synthesis of the cofactor $\mathrm{BH}_{4}$. In earlier times these types of PKU were called malignant PKU, because despite adequate treatment a progressive neurological disease developed. In PKU II dihydropteridine reductase (DHPR) is deficient. (McKusick 26136). Patients lacking DHPR are deficient in the neurotransmitters whose synthesis depends on the normal activitiy of DHPR-dependent tyrosine and tryptophan 


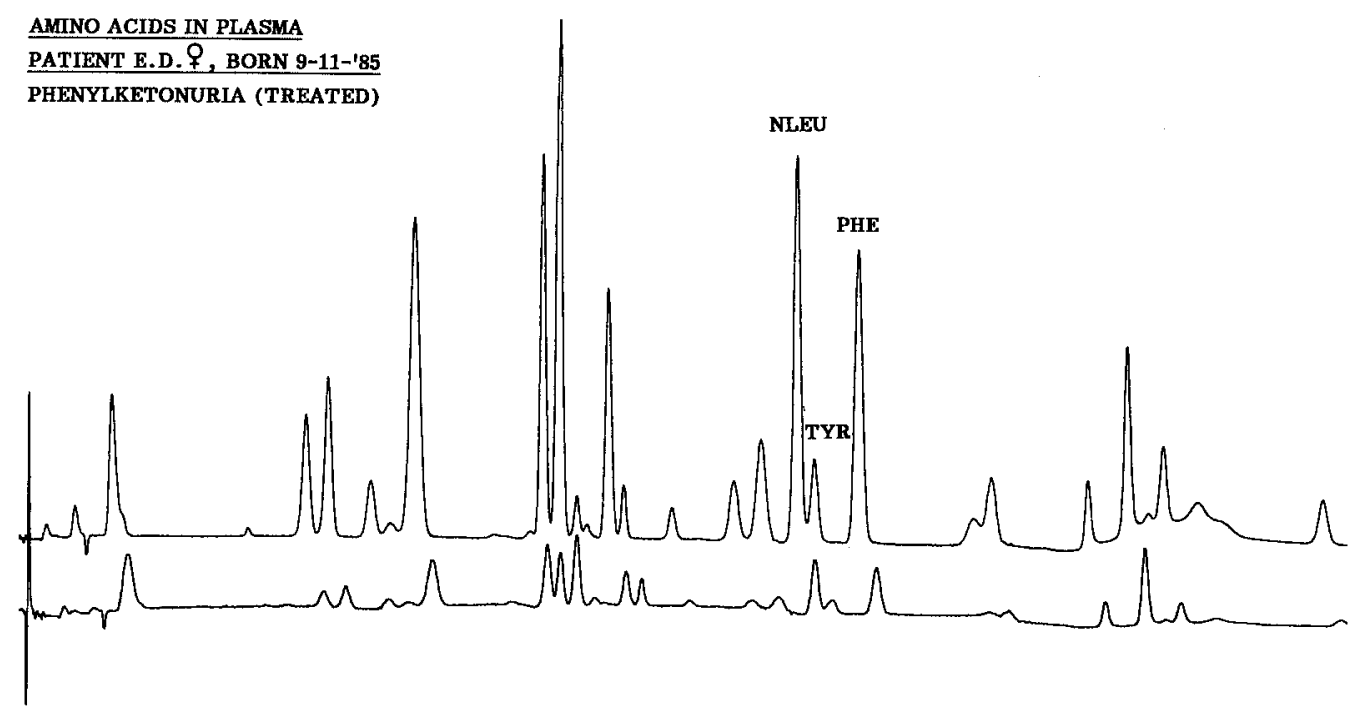

Fig. 30. Chromatogram of amino acids in plasma from a treated patient with PKU

hydroxylases. Treatment of these patients exists in dietary control of the hyperphenylalaninemia, and in restoration of neurotransmitter homeostasis by oral administration of L-dopa, 5-OH-tryptophan, and other agents.

In PKU III (McKusick 26164) enzymes for de novo synthesis of $\mathrm{BH}_{4}$ are deficient: guanosine triphosphate-cyclohydrolase (GTP-CH) and 6-pyruvoyltetrahydropterin synthase (6-PTS). These enzymes catalyze the first two steps in the biosynthesis of $\mathrm{BH}_{4}$ from guanosine triphosphate.

In the Western world in every PKU patient, diagnosed by a neonatal screening test, a $\mathrm{BH}_{4}$ loading test $(2.5-10 \mathrm{mg} / \mathrm{kg})$ is performed. If DHPR, GTP-CH, or 6-PTS is deficient a rapid decrease of the elevated phenylalanine plasma concentration is observed after the $\mathrm{BH}_{4}$ gift. HPLC analysis of pterins will give further evidence for a de novo synthesis defect of $\mathrm{BH}_{4}$.

Another phenomenon in the hyperphenylalanimemia syndromes has become maternal PKU. If the intrapartum maternal phenylalanine level exceeds 1.1 $\mathrm{mmol} / \mathrm{l}$ fetal pathology can occur, leading to intrauterine growth delay, congenital heart malformation, mental retardation and microcephaly. The reason for fetal pathology is that in the fetus the blood phenylalanine level is 1.13-2.91 times higher than in maternal blood. As a consequence it is necessary that the female PKU patient has to use again a PKU diet before she is pregnant. For especially female PKU patients off diet this is often very difficult.

Hyperphenylalaninemia can secondarily occur in the newborn, if the mother is an untreated PKU patient, in primary or secondary tyrosinemia, and in a high neonatal protein intake.

\section{8. $\beta$-Aminoisobutyric acid}

Hyper- $\beta$-aminoisobutyric aciduria is a benign disorder, present in many human populations ( $5-10 \%$ in whites, $40-95 \%$ in Mongolian-derived populations, but not in Asian Indians). (McKusick 21010). The enzyme deficient in this disorder 


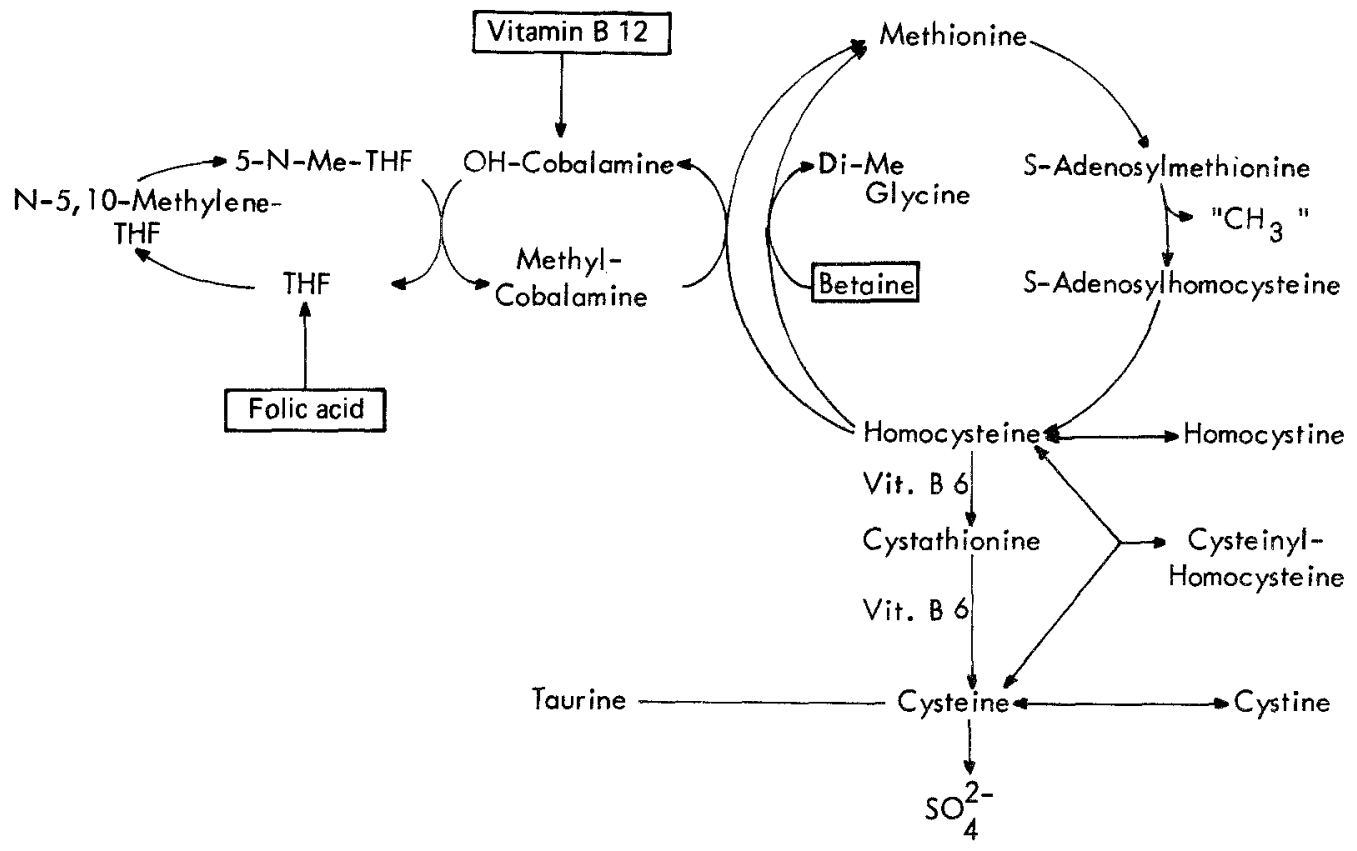

Fig. 31. Metabolic pathway of methionine and homocysteine metabolism

is $\beta$-aminoisobutyrate-pyruvate transaminase. $\beta$-Aminoisobutyric acid is an end product of pyrimidine metabolism. Excessive tissue breakdown results in an activated pyrimidine degradation and as a consequence to a high urinary excretion of $\beta$-aminoisobutyric acid. The same abnormal excretion can be observed in various types of neoplastic disease.

\section{Homocystine}

Homocysteine is normally remethylated for the major part to methionine, in which process folic acid and vitamin $B_{12}$ metabolism plays an important role (see Fig. 31). Remethylating can also occur by use of betaine as a methyl donor, but this is a minor metabolic reaction. Methionine has to be activated to S-adenosylmethionine, to become a strong methyl donor. As a consequence of methylation reactions, S-adenosylhomocysteine is formed, followed by hydrolysis to homocysteine. Homocysteine can escape this cycle by conversion into cystathionine by cystathionine- $\beta$-synthase, with pyridoxalphosphate as a cofactor. Cysteine is formed from cystathionine by a pyridoxalphosphate depending enzyme $\gamma$-cystathioninase. If not rapidly enzymatically converted, homocysteine dimerizes into homocystine, or is coupled to cysteine to form the mixed disulphide cysteinyl-homocysteine.

In classical homocystinuria the conversion of homocysteine into cystathionine is impaired, due to cystathionine- $\beta$-synthase deficiency. (McKusick 23620). There are two types of patients, one type not vitamin $\mathbf{B}_{6}$ dependent, and one type which is vitamin $B_{6}$ dependent. The patients with the vitamin $B_{6}$ independent type, show a variety of clinical abnormalities: 
1. Eye abnormalities (e.g. ectopia lentis, and myopia)

2. Skeletal abnormalities (e.g. osteoporosis, scoliosis, and increased length of long bones)

3. Abnormalities of the central nervous system (e.g. mental retardation, psychiatric disturbances, and seizures)

4. Abnormalities of the vascular system (e.g. vascular occlusions, malar flush, and livedo reticularis)

5. Miscellaneous abnormalities as fair, brittle hair, thin skin, myopathy, and reduced clotting factors

Patients with a mild clinical picture are mostly vitamin $\mathrm{B}_{6}$ dependent. There is high evidence that patients, heterozygote for cystathionine- $\beta$-synthase deficiency, may have thromboembolic abnormalities leading to premature occlusive arterial disease.

Fig. 32 shows the amino acid chromatogram of urine from a patient with classical homocystinuria due to cystahionine- $\beta$-synthase deficiency. Besides homocystine a peak of cysteinyl-homocysteine is also detectable.

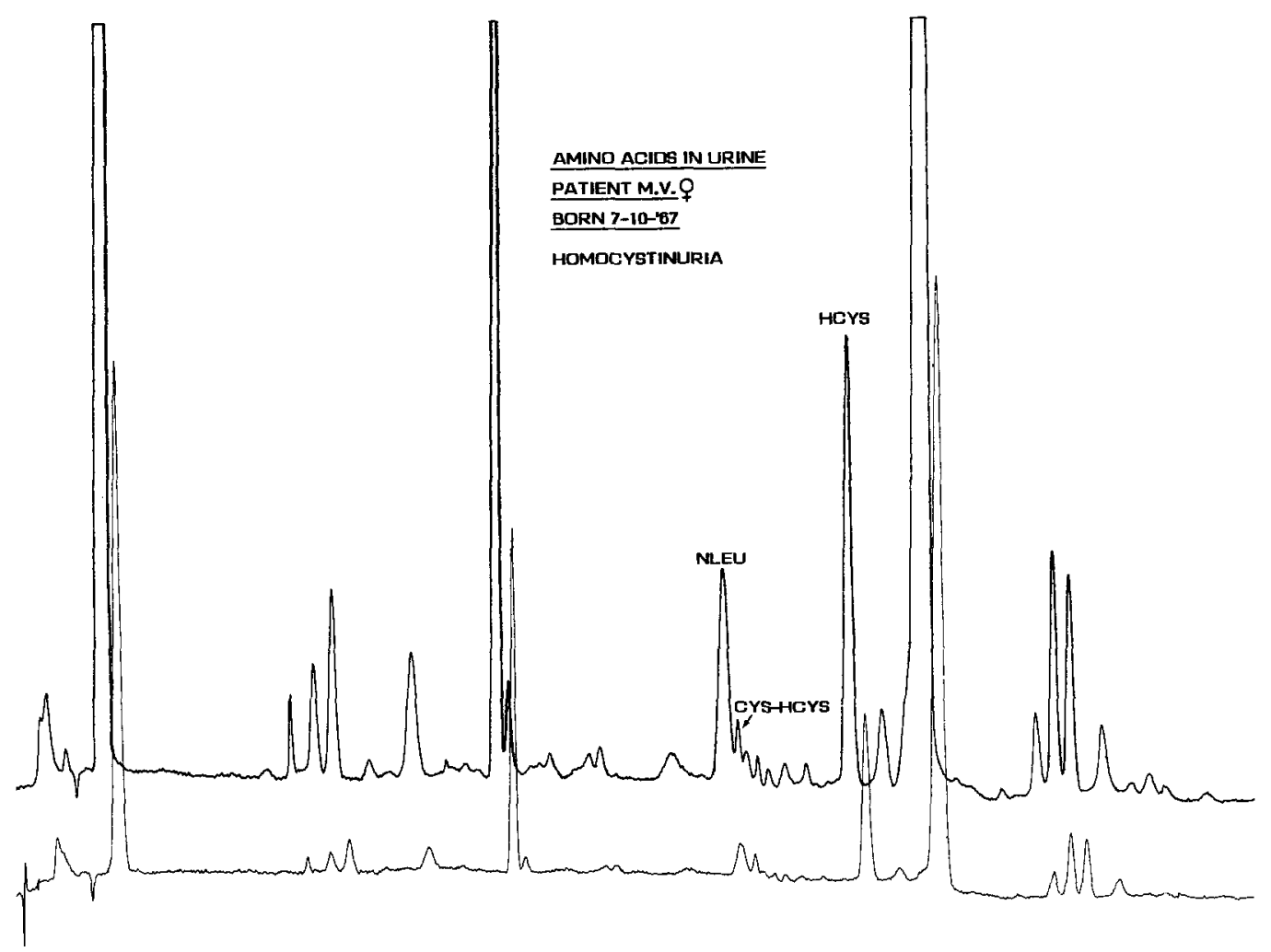

Fig. 32. Chromatogram of amino acids in urine from a patient with homocystinuria due to cystathionine- $\beta$-synthase deficiency

There are other causes for homocystinuria, which will be summarized:

1. Severe vitamin $B_{6}$ deficiency

2. Impaired activity of 5-methyltetrahydrofolate-homocysteine methyltransfe- 
rase, due to:

a. 5,10-Methylenetetrahydrofolate reductase deficiency (McKusick 23625)

b. Folate deficiency

c. Nutritional vitamin $B_{12}$ deficiency

d. Defective absorption of vitamin $B_{12}$

e. Failure to convert cobalamin to methyl- or adenosylcobalamine

f. Failure to accumulate methylcobalamin (McKusick 23627)

3. Administration of 6-azauridine triacetate, or isonicotinic acid hydrazide

4. Bacterial decomposition of urinary cystathionine.

An example of the amino acid chromatogram in urine from a patient with homocystinuria due to 5,10-methylenetetrahydrofolate reductase deficiency is given in Fig. 33. It is striking that in this urine cystine and cysteinyl-homocysteine are markedly increased, whereas homocystine is hardly detectable.

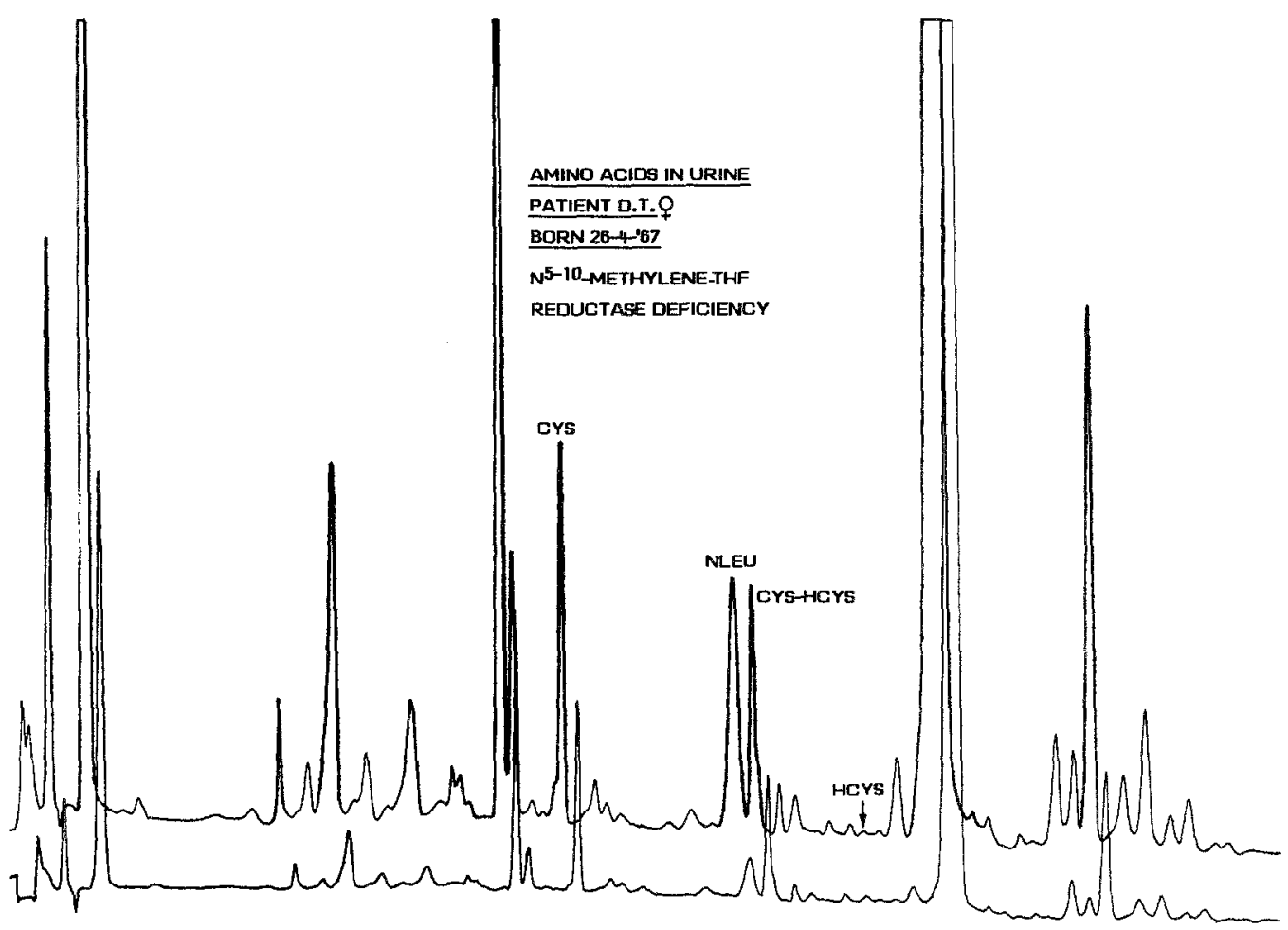

Fig. 33. Chromatogram of amino acids in urine from a patient with homocystinuria due to 5,10 -methylene-THF reductase deficiency

30. $\gamma$-Aminobutyric acid

There are three primary disorders of $\gamma$-aminobutyric acid metabolism, but these inborn errors are not expressed in $\gamma$-aminobutyric aciduria. $\gamma$-Aminobutyric acid (GABA) can be detectable in urine in $\beta$-alanine- $\alpha$-ketoglutarate transaminase deficiency (see $26 . \beta$-alanine), and as a result of intestinal dysbacteriosis. 


\section{Tryptophan}

Tryptophan is almost totally destroyed in an amino acid analyser. To estimate tryptophan in body fluids HPLC methods have to be used. Tryptophan excretion in urine can be increased in Hartnup disease (McKusick 23450), and due to hypoalbuminemia. Urinary tryptophan excretion can be decreased due to chronic diarrhea or constipation.

\section{Ethanolamine}

Although ethanolamine is not an amino acid, ethanolamine is always present in the amino acid chromatogram of urine. Primary ethanolaminuria (McKusick 22715 ) is described in two siblings, with cardiomegaly, generalized muscular hypotonia, cerebral dysfunction and death at ages 10 and 17 months. In liver ethanolamine kinase deficiency was demonstrated. Urinary ethanolamine can secondarily be increased in newborns, and in liver cirrhosis.

\section{Ornithine}

Ornithine is an intermediate in the urea cycle. Ornithine transcarbamylase (OTC) deficiency is a primary disorder of ornithine metabolism, not leading to excessive aberrations in plasma or urine concentrations (see under 15. hyperammonemia syndromes). Ornithine does not accumulate in OTC deficiency, because ornithine can escape the urea cycle by $\delta$-transamination (see Fig. 16), or by a decarboxylation reaction to putrescine. Catalyzed by the pyridoxalphosphate requiring enzyme ornithine- $\delta$-aminotransferase (OAT), ornithine can be converted into glutamic- $\delta$-semialdehyde (see step 3 in Fig. 10). Glutamic- $\delta$ semialdehyde can be oxidized to glutamic acid (see step 5 in Fig. 10), or cyclizes spontaneously to $\Delta$-pyrroline-5-carboxylic acid (see step 6 in Fig. 10).

OAT deficiency with hyperornithinemia and hyperornithinuria is found in patients with gyrate atrophy of the choroid and retina. (McKusick 25887). These patients have mostly a slowly progressive loss of vision, usually leading to blindness in the fourth decade of life. The first symptoms are myopia and decreased night vision, followed by reduced peripheral vision with constriction of visual fields, development of posterior subcapsular cataracts, and total blindness. About half of the patients described with OAT deficiency are Finnish.

Patients with OAT deficiency have ornithinuria and 10- to 20-fold elevations of ornithine in plasma, CSF, and aqueous humor. Fig. 34 shows the amino acid chromatogram in urine from a patient with OAT deficiency. It is seen from the figure that also lysine is markedly elevated, whereas cystine and arginine are moderately increased. In addition to an abnormal urinary amino acid excretion pattern, abnormal excretion of the $\delta$-lactam of ornithine (3-amino-piperid-2one), the methylester of ornithine, and $\gamma$-glutamylornithine are documented.

As OAT is a pyridoxalphosphate depending enzyme, some patients have shown biochemical improvements on vitamin $B_{6}$ therapy. Clinical improvements are not striking; it might be that the progession of the disease is slower.

Another disease, in which ornithine is involved, is the hyperornithinemiahyperammonemia-homocitrullinuria (HHH) syndrome. (McKusick 23897). 


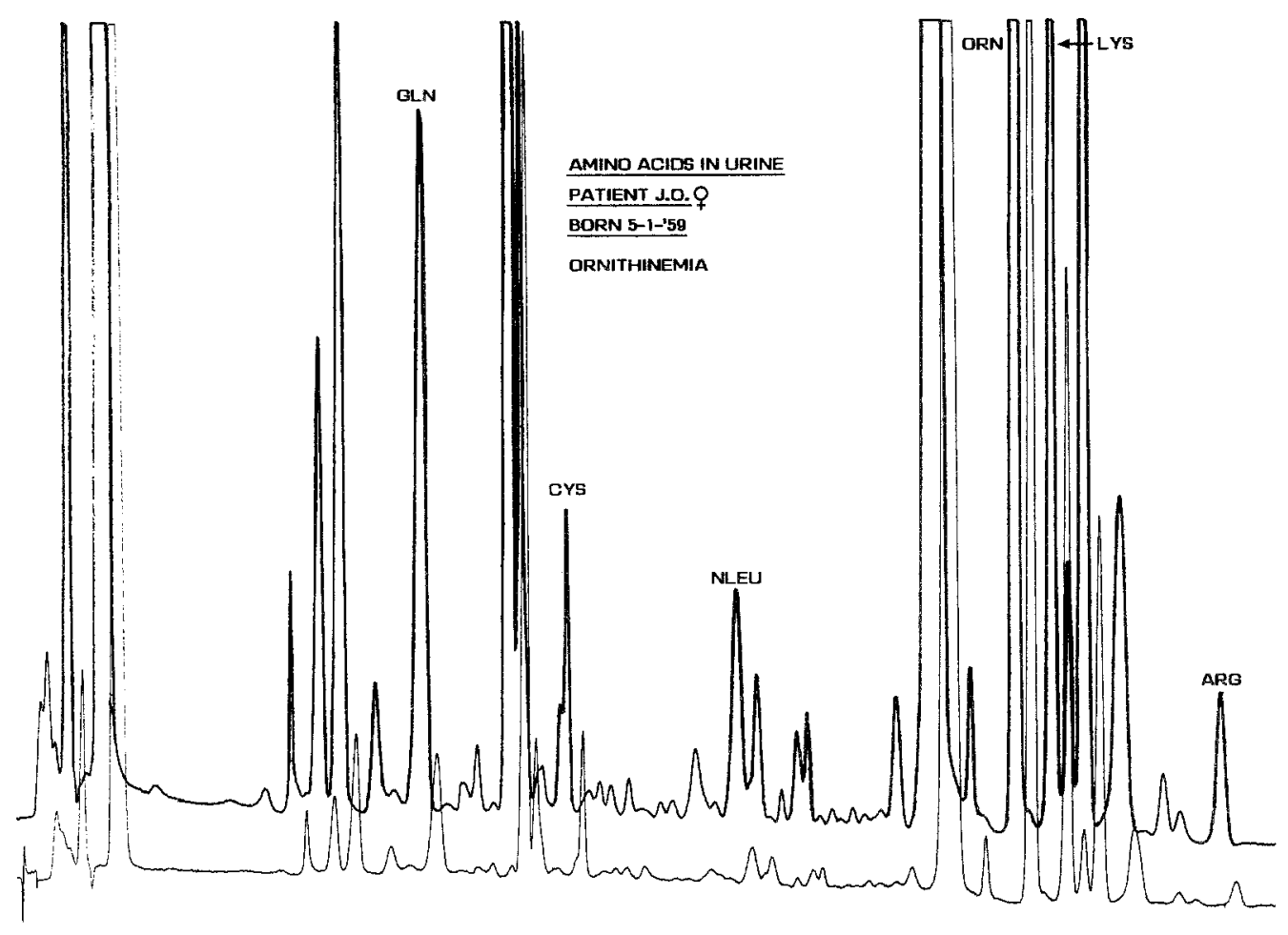

Fig. 34. Chromatogram of amino acids in urine from a patient with ornithine- $\delta$-aminotransferase deficiency

Most patients have a histories of intermittent hyperammonemia. They refuse to eat and have vomiting, lethargy, or coma, when fed high-protein formulas, milk or meat. Growth is inadequate and there is a developmental delay with low normal or severe mental retardation. Some patients have an increased bleeding tendency.

No enzyme deficiency has been detected. Besides hyperornithinemia, hyperammonemia and homocitrullinuria the patients show mild ornithinuria, and an increased urinary excretion of 3-amino-piperid-2-one, $\gamma$-glutamylornithine, orotic acid, and polyamines.

Ornithine excretion in urine is elevated in cystinuria with dibasic amino aciduria as described under 19. cystine.

\section{Lysine}

The metabolic pathway of lysine degradation is shown in Fig. 7. The first two steps are catalyzed by a bifunctional protein $\alpha$-aminoadipic semialdehyde synthase. The protein associates two enzyme activities: lysine- $\alpha$-ketoglutarate reductase and saccharopine dehydrogenase. In patients with (familial) hyperlysinemia, a deficiency of lysine-a-ketoglutarate reductase together with a deficiency of saccharopine dehydrogenase is measured, indicating an impairment of the bifunctional protein. (McKusick 23870). The clinical picture is heterogeneous: some patients have severe mental retardation, others are symptom free. 
We were involved in two siblings with hyperlysinemia. Both patients had protein aversion, were extremely vomiting on a normal protein diet, and were severely mentally retarded. On a protein restricted diet protein aversion and vomiting disappeared. The younger sister, diagnosed earlier than her brother, is less mentally retarded. Fig. 35 shows the amino acid chromatogram in urine of the younger sister with hyperlysinuria (and hyperlysinemia). In the chromatogram homocitrulline and arginine are mildly elevated.

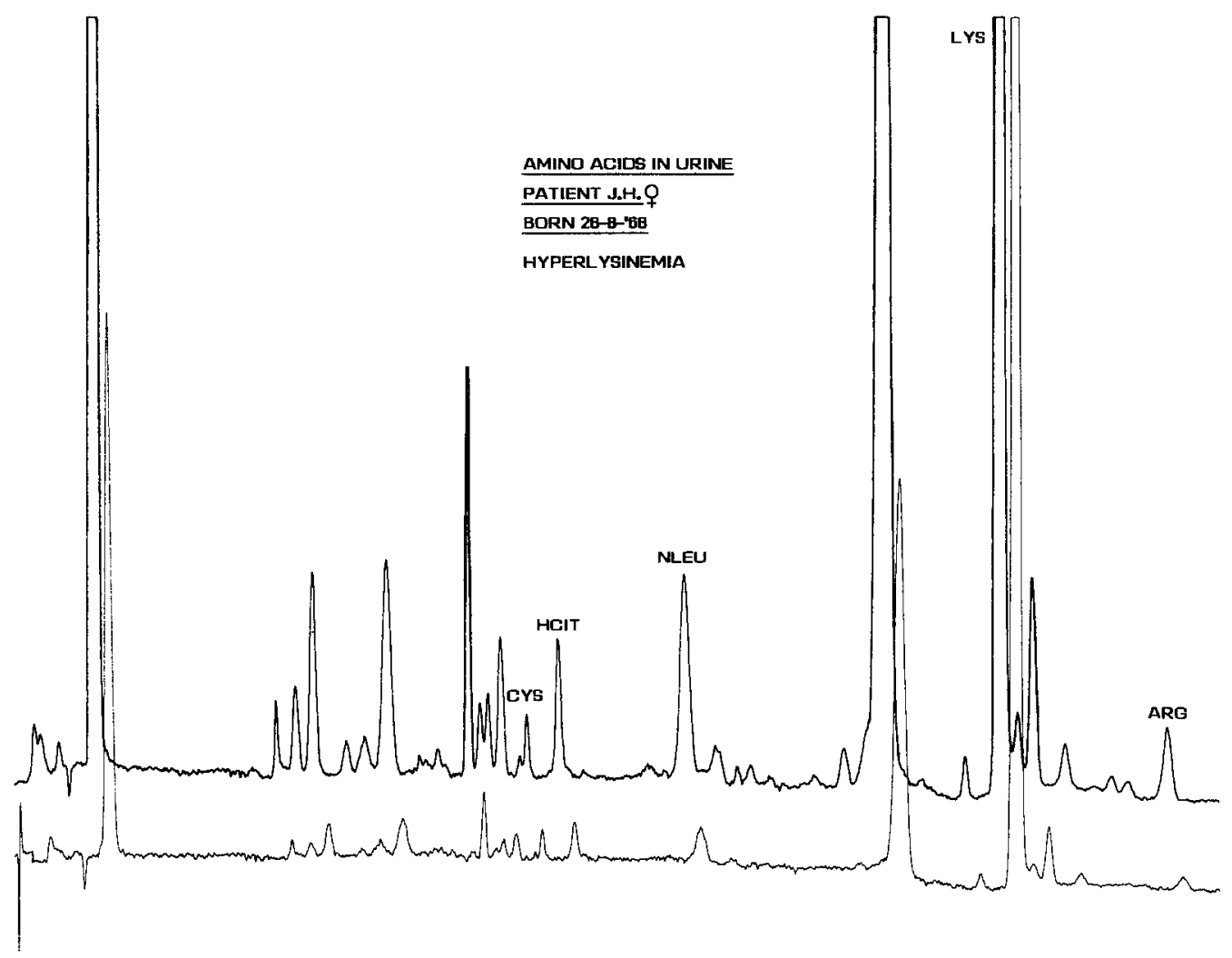

Fig. 35. Chromatogram of amino acids in urine from a patient with lysine- $\alpha$-ketoglutarate reductase deficiency due to a defect of the bifunctional protein $\alpha$-amino-adipic semialdehyde synthase

Hyperlysinemia and -uria is also reported in patients with saccharopinuria. (McKusick 26870). In these patients saccharopine dehydrogenase is more deficient than lysine- $\alpha$-ketoglutarate reductase. The first reported patient was 22 years of age, moderately retarded with EEG abnormalities, but without seizures. In addition to lysine and saccharopine, urinary excretion of citrulline and histidine was also elevated. A second patient had spastic diplegia, but somatically and mentally she was normal.

Membrane transport of the dibasic amino acids ornithine, lysine and arginine is abnormal in four specific diseases:

1. Classical cystinuria (see under 19. cystinuria).

2. Hyperdibasic aminoaciduria type I, without hyperammonemia. (McKusick 
22269). In 13 members of a French-Canadian kindred excessive urinary lysine, ornithine and arginine excretion was discovered. Endogenous renal clearance of the three dibasic amino acids was increased, but that of cystine was normal. The patients were asymptomatic. Only the proband had a mild intestinal malabsorption syndrome. Another reported patient was mentally retarded. In four generations of the family heterozygote excretion values of the dibasic amino acids were found.

3. Hyperdibasic aminoaciduria type II, with hyperammonemia. (McKusick 22270). This disorder is known as lysinuric protein intolerance (LPI). The patients do not have symptoms when breast-fed, but develop protein aversion, vomiting and diarrhea after weaning. If they are forced to high protein intake, they may come in coma. During and after infancy they develop growth retardation, hepatosplenomegaly, muscle hypotonia, sparse hair, and osteoporosis. Most patients are mentally normal, some are moderately retarded. LPI is particularly frequent in Finland. The exact cause of LPI is unknown. In vivo and in vitro intestinal and renal studies strongly suggest a transport defect of dibasic amino acids, localized in the basolateral (antiluminal) membrane of the epithelial cells.

Plasma lysine, ornithine and arginine levels are low normal. In urine lysine excretion is strongly increased, whereas urinary ornithine and arginine excretion is moderately elevated. As an indication for hyperammonemia, alanine and glutamine can be increased in plasma and urine, but a significant

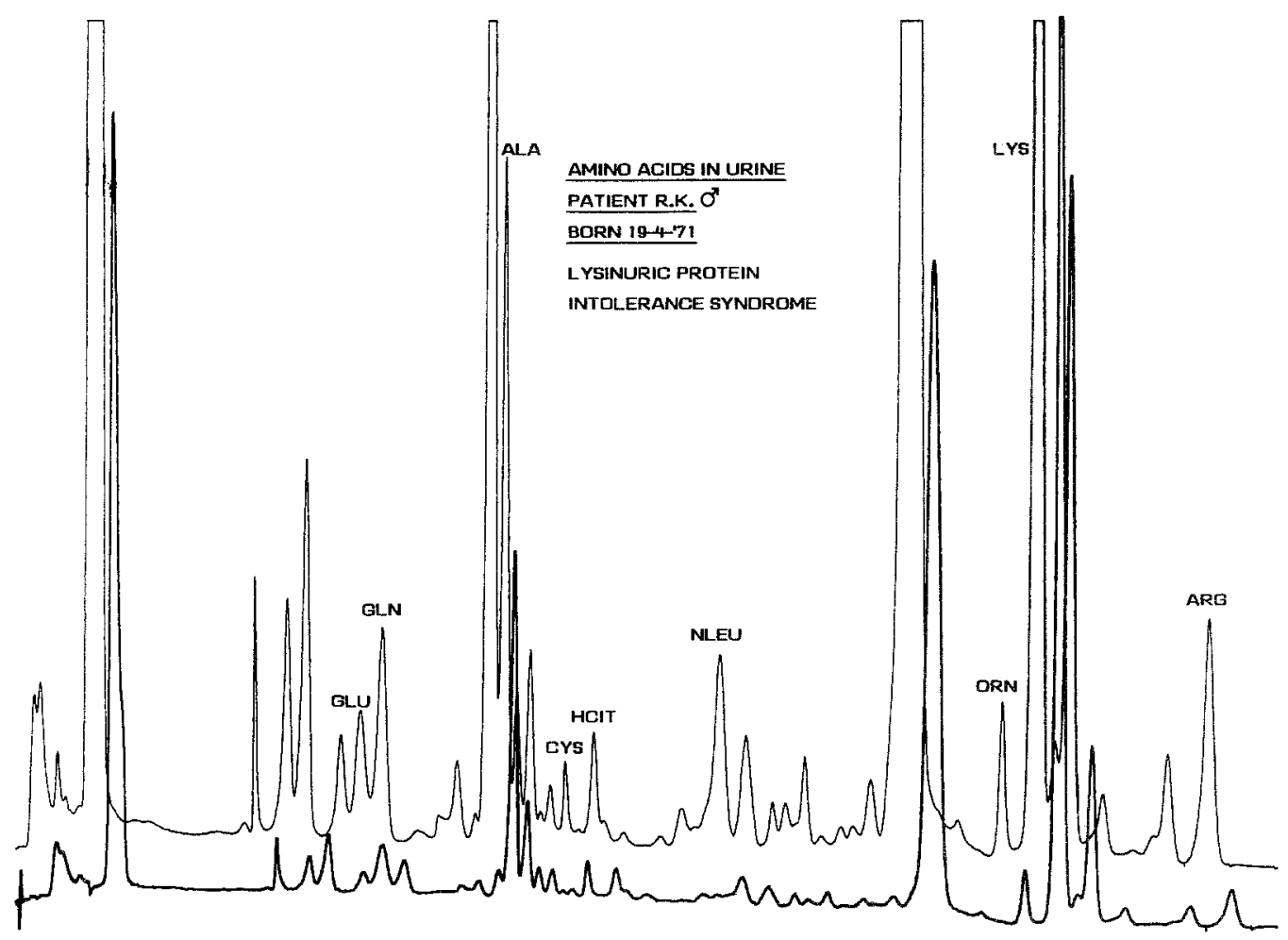

Fig. 36. Chromatogram of amino acids in urine from a patient with lysinuric protein intolerance syndrome 
orotic aciduria is more characteristic. In plasma citrulline concentration is elevated. Fig. 36 shows the amino acid excretion pattern in urine from a patient with LPI.

4. Lysine malabsorption or isolated lysinuria. (McKusick 24795). One Japanese patient was described with severe mental retardation, convulsions, growth failure, and signs of malnutrition. Only intestinal lysine absorption was decreased and there was isolated lysinuria. Blood ammonia was always normal.

Urinary lysine and cystine concentration can secondarily be increased in newborns during the first months of life. Lysine excretion can be elevated in hyperammonemia syndromes.

\section{1-Methylhistidine}

No primary disorder of 1-methylhistidine metabolism is reported. 1-Methylhistidine is synthesized from histidine, and can be liberated by hydrolysis of anserine (see Fig. 37). Urinary 1-methylhistidine can secondarily be increased due to renal insufficiency, and by alimentary cause (chicken meat).

\section{Histidine}

Pathways of histidine catabolism are given in Fig. 37. The most important pathway of histidine degradation is through urocanic acid. A primary disorder of histidine metabolism is histidinemia, due to histidase deficiency, which affects the conversion of histidine into urocanic acid. (McKusick 23580). It is generally accepted now that histidinemia is a benign metabolic disorder. As a result of histidine accumulation other degradation reactions are activated, leading to secondary elevated urinary excretion of imidazolepyruvic acid, imidazolelactic acid, and imidazoleacetic acid. These metabolites can be detected in urine by thin-layer chromatography of imidazoles, or by HPLC. Fig. 38 shows the amino acid chromatogram in urine from a patient with histidinemia.

In a few patients isolated histidinuria without histidinemia is described. (McKusick 23583). Intestinal and renal tubular transport of histidine are impaired. Mental retardation or myoclonic seizures were associated clinical symptoms.

Urinary histidine excretion can secondarily be increased in newborns, and in folic acid deficiency.

\section{3-Methylhistidine}

No primary defect in 3-methylhistidine metabolism is described. 3-Methylhistidine is synthesized out of histidine. Secondarily 3-methylhistidine can be increased in urine by malnutrition, or starvation.

\section{Carnosine and anserine}

Carnosine is a dipeptide: $\beta$-alanyl-L-histidine (see Fig. 37). In humans carnosine is hydrolyzed by tissue carnosinase and serum carnosinase. Patients have been 


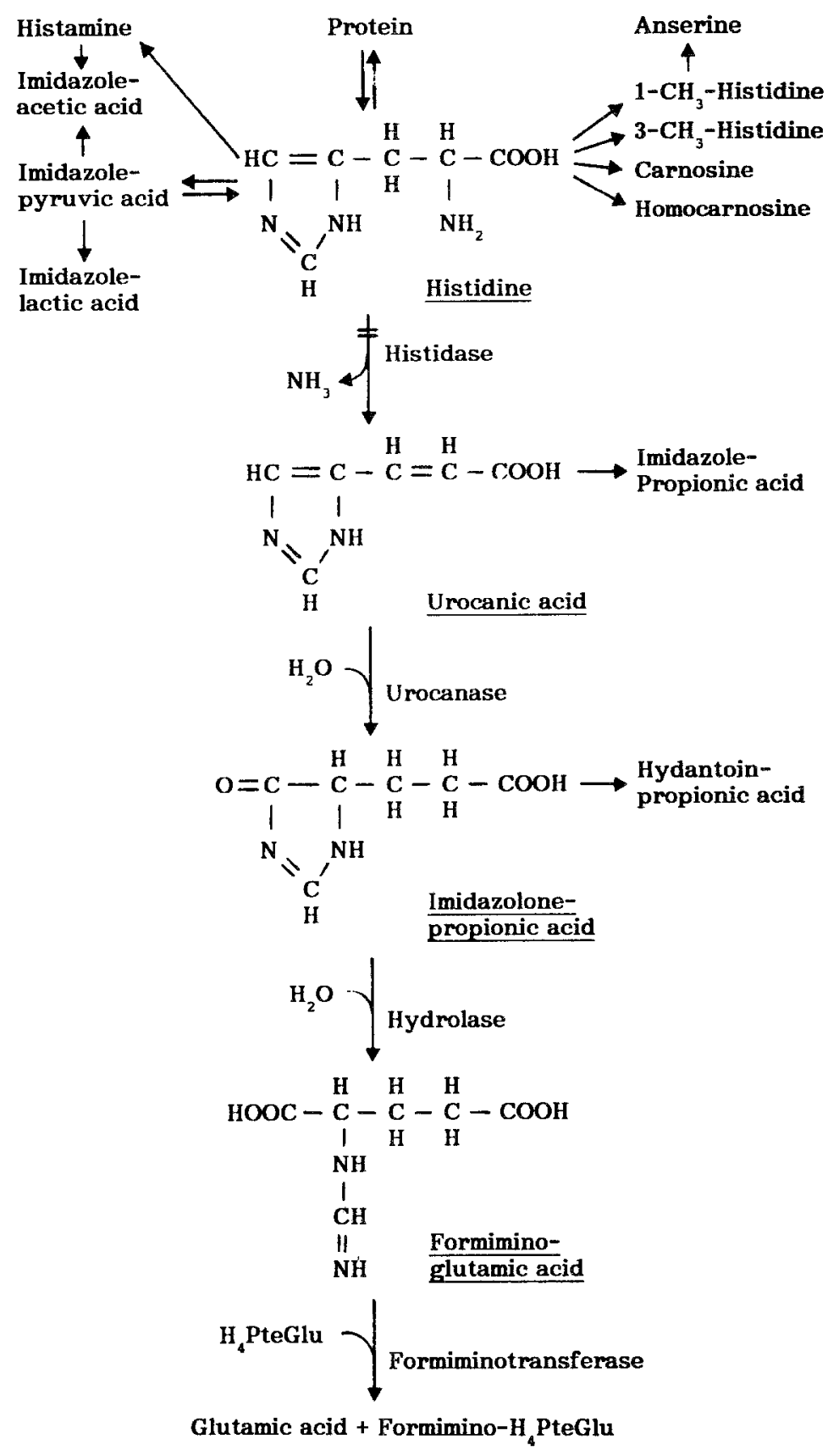

Fig. 37. Metabolic pathway of histidine degradation

described with carnosinuria due to serum carnosinase deficiency. (McKusick 21220). Patients have neurological symptoms, like myoclonic seizures, and psychomotor retardation. In young infants up to 2 years of age serum carnosinase activity is low. In patients with persistent carnosinuria due to serum carnosinase deficiency the dipeptide anserine ( $\beta$-alanine-1-methyl-histidine) is also elevated in urine. Anserine has an alimentary origin (chicken, special kinds of meat), and is normally also hydrolyzed by serum carnosinase. 


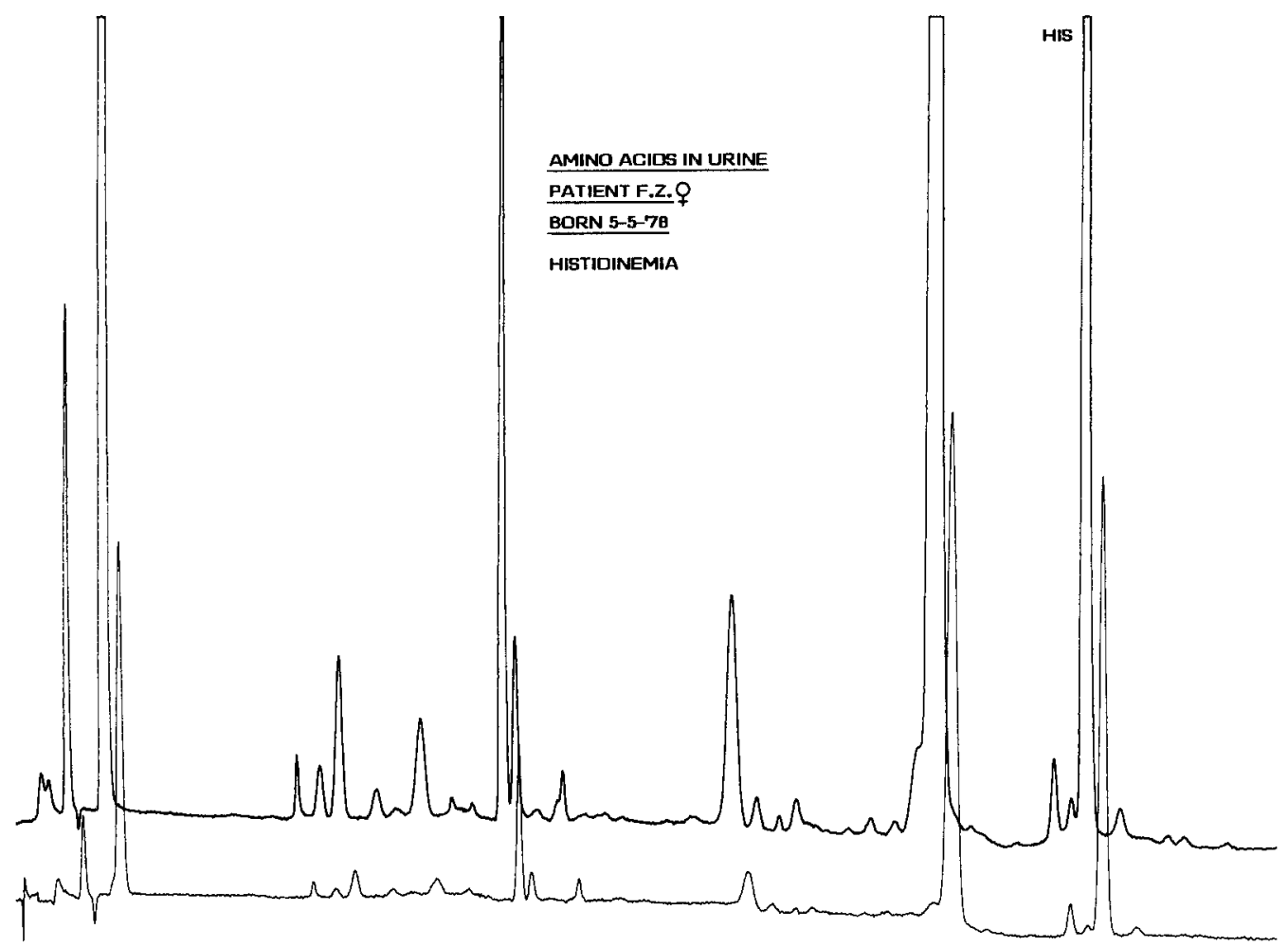

Fig. 38. Chromatogram of amino acids in urine from a patient with histidase deficiency

\section{Arginine}

A primary disorder of arginine metabolism has been described above under 15. hyperammonemia syndromes. Arginine transport is defective in classical cystinuria (see 19. cystinuria), and in dibasic aminoaciduria type I and II (see 34. lysine). Urinary arginine excretion is secondarily increased in ornithinemia (see 33. ornithine) and in hyperlysinemia (see 34. lysine).

\section{Conclusions}

An dedicated amino acid analyzer remains an important tool in the diagnosis of aminoacidopathies. Inspite of numerous attempts to use modern HPLC methods to analyze amino acids in physiological fluids, we still belief that in the metabolic field, there is no alternative for the dedicated amino acid analyzer.

Greater experience with the inborn errors of amino acid metabolism has led to increased evidence for genetic heterogeneity, which is confirmed by results of recombinant DNA technology [6]. Many disorders are now known to contain clinically and biochemically apparent subgroups. Genetic disorders need to be distinguished from acquired abnormalities. We have tried to make a contribution to the differential diagnosis of (inherited) amino acid metabolism or transport disorders. 


\section{References}

1. Blom W, Polder-Mol AC, Kelholt-Dijkman HH, Hierck L, Huijmans JGM (1990) J Inher Metab Dis 13: 315-320

2. Bremer HJ, Duran M, Kamerling JP, Pzryrembel H, Wadman SK (eds) (1981) Disturbances of amino acid metabolism: Clinical chemistry and diagnosis. Urban \& Schwarzenberg, Baltimore Munich

3. Nyhan WL (ed) (1984) Abnormalities in amino acid metabolism in clinical medicine. Appleton-Century-Crofts, Norwalk, Conn

4. Holton JB (ed) (1987) The inherited metabolic diseases. Churchill Livingstone, Edinburgh

5. Fernandes J, Saudubray JM, Tada K, (eds) (1990) Inborn metabolic disease, diagnosis and treatment. Springer, Berlin Heidelberg New York Tokyo

6. Scriver CR, Beaudet AL, Sly WS, Valle D (eds) (1989) The metabolic basis of inherited disease. McGraw-Hill, New York

7. McKusick VA (1988) Mendelian inheritance in man, 8th edn. The John Hopkins University Press, Baltimore London

8. Teijema HL, Van Gelderen HH, Giesberts MAH, Laurent de Angulo MSL (1974) Metabolism 23: 115-123

9. Blom W, Fernandes J (1979) Clin Chim Acta 91: 117-125

10. Fisher MH, Gerritsen T, Opitz JM (1974) Humangenetik 24: 265-270

11. Fisher MH, Brown RR (1980) Am J Med Genet 5: 35-42

12. Kleijer WJ, Blom W, Huijmans JGM, Mooyman MCT, Berger R, Niermeijer MF (1984) Prenatal Diagnosis 4: 113-118

13. Blom W, Huijmans JGM, Van den Berg GB (1989) J Inher Metab Dis 12 [Suppl 1]: 64-88

14. Marescau B, De Deyn PP, Lowenthal A (1990) Pediatr Res 27: 297-303

15. Wada Y, Tada K, Minagawa A, Yoshida T, Morikawa T, Okamura T (1963) Tohoku J Exp Med 81: 46-55

16. Brodehl J, Gellissen K, Kowalewski S (1967) Klin Wochenschr 45: 38-40

17. Jeune M, Collombel C, Michel M, David M, Guibaud P, Guerrier G, Albert J (1970) Ann Pediatr 17: 85-93

18. Pollitt RJ, Green A, Smith R (1985) J Inher Metab Dis 8: 75-79

Authors' address: Dr. W. Blom, Metabolic Laboratory, Department of Pediatrics, Sophia Children's Hospital, Erasmus University Rotterdam, Gordelweg 160, NL-3038 GE Rotterdam, The Netherlands. 